M. Itô

Nagoya Math. J.

Vol. 50 (1973), 117-148

\title{
NOYAUX RÉGULIERS ET NOYAUX SINGULIERS
}

\author{
MASAYUKI ITÔ
}

\section{Introduction}

Dans l'article précédent [6], nous définissions deux classes des noyaux de convolution symétriques sur un groupe abélien localement compact et dénombrable à l'infini; l'une est régulière et l'autre est singulière. L'article [6] est principalement consacré à la démonstration du théorème suivant:

Soient $N$ et $N^{\prime}$ respectivement un noyau de convolution régulier et un noyau de convolution singulier; alors pour que $N+N^{\prime}$ satisfasse au principe de domination, il faut et il suffit que $N$ soit un noyau de convolution de Dirichlet et que $N^{\prime}$ soit périodique à tout le point du support de $N$ et satisfasse aussi au principe de domination. Par conséquent, en particulier si $N$ est partout dense dans $X, N^{\prime}$ est constant.

Remarquons que pour les noyaux de convolution symétriques, le principe de domination et le principe complet $\mathrm{du}$ maximum sont équivalents. On obtient facilement que si, pour un noyau de convolution régulier $N$ et pour un noyau de convolution singulier $N^{\prime}, N+N^{\prime}$ satisfait au principe de domination, alors $N_{p}+N^{\prime}$ y satisfait automatiquement, où $\left(N_{p}\right)$ est la résolvante associée au noyau $N$.

Soit $X$ un espace localement compact, non-compact et à base dénombrable; supposons toujours qu'il existe une mesure de Radon positive $\xi$ partout dense dans $X$, c'est-à-dire, quel que soit $\omega$ un ouvert non-vide de $X, \xi(\omega)>0$. On fixera, dès maitenant, $X$ et $\xi$.

Un noyau $N$ relatif à $X$ et à $\xi^{(1)}$ est dit régulier s'il existe l'espace fonctionnel faiblement régulier $H(N)$ au noyau $N$ (voir [3] et [5]). Un noyau symétrique $N^{\prime}$ est, par définition, singulier si l'on a $L^{2}(\xi) \cap H\left(N^{\prime}\right)$ $=\{0\}$.

Remarquons ici que si un noyau relatif à $X$ et à $\xi$ est symétrique

Received February 4, 1972.

(1) Voir [7]. 
et de type positif, il existe toujours l'espace fonctionnel $H(N)$ au noyau $N$ (voir [5]).

On montrera d'abord que si un noyau symétrique $N$ satisfait au principe de domination, alors on peut écrire $N=N_{0}+N^{\prime}$, où $N_{0}$ est un noyau régulier satisfaisant au principe de domination et $N^{\prime}$ est un noyau singulier. On discutera ensuite quand $N^{\prime}$ est un noyau $\left(p N_{p}\right)$-invariant, où $\left(N_{p}\right)_{p>0}$ la résolvante associée à $N_{0}$. Finalement, on obtiendra le résultat suivant:

Soient $N$ et $N^{\prime}$ respectivement un noyau régulier satisfaisant au principe complet du maximum et un noyau singulier. Si, quel que soit $p>0, N_{p}+N^{\prime}$ satisfait au principe complet du maximum, alors, quelle que soit $f$ de $M_{K}$, on a, pour $\xi$-presque tout $x$ de $X, N^{\prime} f(x)=N^{\prime} f(y)$ $\xi$-p.p. sur $\operatorname{Supp}\left(N ; \varepsilon_{x}\right)$, où $\left(N_{p}\right)_{p>0}$ est la résolvante associée au noyau $N, N^{\prime} f$ est le potentiel de $f$ par rapport à $N^{\prime}$ et $\operatorname{Supp}\left(N ; \varepsilon_{x}\right)$ est le support du $N$-potentiel de la mesure de Dirac $\varepsilon_{x}$ à $x$.

\section{Préliminaires}

On note $E$ la $\sigma$-algèbre constituée par tous les ensembles $\xi$-mesurables de $X$ et on écrit $E_{0}=\{e \in E ; \bar{e}$ : compact $\}$.

Un noyau relatif à $X$ et à $\xi$ est, par définition, une application $N$ de $E \times E$ à $[0,+\infty]$ telle que, quel que soit $e_{0}$ de $E_{0}, e \rightarrow N\left(e, e_{0}\right)$ et $e \rightarrow N\left(e_{0}, e\right)$ soient complètement additives sur $E$ et absolument continues par rapport à $\xi$. Cela était premièrement défini dans [7]. Si, quels que soient $e_{1}, e_{2}$ de $E, N\left(e_{1}, e_{2}\right)=N\left(e_{2}, e_{1}\right)$, alors $N$ est dit symétrique. On dit que $N$ est de type positif si, quels que soient $e_{1}, e_{2}$ de $E_{0}$,

$$
\frac{N\left(e_{1}, e_{2}\right)+N\left(e_{2}, e_{1}\right)}{2} \leqq\left(N\left(e_{1}, e_{1}\right)\right)^{1 / 2}\left(N\left(e_{2}, e_{2}\right)\right)^{1 / 2}
$$

Il est évident que tout le noyau relatif à $X$ et à $\xi$ est une mesure de Radon positive dans l'espace produit $X \times X$ (cf. par exemple, [8]).

Soit $N$ un noyau relatif à $X$ et à $\xi$; pour une fonction $f \xi$-mesurable et non-négative dans $X$, et pour un ensemble $e$ de $E_{0}$, l'intégrale

$$
N(e, f)=\int f(y) N(e, d y)
$$

a un sens. Si l'application $e \rightarrow N(e, f)$ est complètement additive sur $E$ et absolument continue par rapport à $\xi$, sa densité s'écrit $N f$. Pour une fonction $f$ réelle et $\xi$-mesurable dans $X, N f=N f^{+}-N f^{-}$s'appelle 
le potentiel de $f$ par rapport au noyau $N$ dès que $N f^{+}$et $N f^{-}$sont définis. Notons $M_{K}=M_{K}(X)$ l'ensemble des fonctions bornées et $\xi$ mesurables dans $X$ à valeurs réelles et à support compact; alors, pour tout noyau $N$ relatif à $X$ et à $\xi$ et pour toute fonction $f$ de $M_{K}, N f$ a un sens.

On dit que $N$ satisfait au principe de domination (resp. au principe complet du maximum) si, quelles que soient $f, g$ de $M_{K}^{+}=\left\{f \in M_{K} ; f \geqq 0\right\}$, $N f(x) \leqq N g(x)$ (resp. $N f(x) \leqq N g(x)+1$ ) presque partout pour $\xi$ (noté simplement p.p.) sur $X$ dès que la même inégalité a lieu p.p. sur $\operatorname{Supp}(f)$. On désigne par $\operatorname{Supp}(f)$ le support de la mesure $f \xi$.

Pour un noyau symétrique $N$ de type positif, l'ensemble $\left\{N f ; f \in M_{K}\right\}$ est un espace pré-hilbertien par le produit scalaire

$$
(N f, N g)=\int N f(x) g(x) d \xi(x) \text {. }
$$

Sa complété est un seul espace hilbertien $H=H(N)$ des fonctions réelles et localement $\xi$-sommables dans $X$ et telle que, quelles que soient $f$ de $M_{K}$ et $u$ de $H$,

$$
(N f, u)=\int u(x) f(x) d \xi(x)
$$

Evidemment $H$ vérifie la condition suivante:

(A) A un compact donné $K$ de $X$, on peut associer une constante $A(K)>0$ telle que, quelle que soit $u$ de $H(N)$,

$$
\int_{K}|u(x)| d \xi(x) \leqq A(K)\|u\|,
$$

où $\|u\|$ est la norme de $u$ dans $H(N)$.

En général, un espace fonctionnel (relatif à $X$ et à $\xi$ ) est, par définition, un espace hilbertien constitué par fonctions réelles et localement $\xi$-sommables dans $X$ et vérifiant la condition (A) (cf. [1] et [3]). Donc $H(N)$ s'appelle l'espace fonctionnel au noyau $N$. Soit $H$ un espace fonctionnel; alors, d'après la condition (A), pour une fonction $f \operatorname{de} M_{K}$, il existe une fonction $u_{f}$ de $H$, et une seule telle que, quelle que soit $u$ de $H$,

$$
\left(u_{f}, u\right)=\int u(x) f(x) d \xi(x)
$$

On appelle $u_{f}$ le potentiel de $f$ dans $H$. En particulier, si $u_{f} \geqq 0$ dès 
que $f \geqq 0$, il existe alors un noyau symétrique $N$ de type positif, et un seul tel que, quelle que soit $f$ de $M_{K}, u_{f}=N f$.

Soient $N_{1}$ et $N_{2}$ deux noyaux symétriques relatifs à $X$ et à $\xi$; alors pour que

$$
N_{1} N_{2}: E \times E \ni\left(e_{1}, e_{2}\right) \rightarrow \int N_{1} c_{1}(x) N_{2} c_{2}(x) d \xi(x)
$$

soit un noyau relatif à $X$ et à $\xi$, où $c_{i}(i=1,2)$ est la fonction caractéristique de $e_{i}$, il faut et il suffit que, quelle que soit $f$ de $M_{K}$,

$$
\int\left|N_{1} f(x) N_{2} f(x)\right| d \xi(x)<+\infty .
$$

Si $N_{1} N_{2}$ a un sens, cela s'appelle la composée de $N_{1}$ et de $N_{2}$. Voir, par exemple, [7].

Il est déjà connu qu'à un noyau symétrique $N$ relatif à $X$ et à $\xi$, on peut associer une famille $\left(N_{p}\right)_{p>0}$ des noyaux relatifs à $X$ et à $\xi$ et qui vérifie

"Quels que soient $p>0$ et $q>0$,

$N_{p}-N_{q}=(q-p) N_{p} N_{q}$ et $\lim _{p \rightarrow 0} N_{p}\left(e_{1}, e_{2}\right)=N\left(e_{1}, e_{2}\right)$ sur $E \times E$ ',

si et seulement si $N$ satisfait au principe de domination et $N$ est régulier (cf. [5]).

Si $\left(N_{p}\right)_{p>0}$ existe, elle est uniquement déterminée et s'appelle la résolvante associée au noyau $N$. On rappelle que si $H(N) \cap L^{2}(\xi)$ est dense dans $H(N), N$ est dit régulier (voir aussi [5]).

Rappelons aussi que si un noyau symétrique $N$ satisfait au principe de domination, alors $N$ est de type positif, d'où il existe l'espace fonctionnel au noyau $N$, et que s'il existe la résolvante associée au noyau $N$, alors $N$ satisfait au principe de domination (voir [7]).

$L^{2}(\xi)$ est évidemment un espace fonctionnel au noyau positif, et son noyau $U$ s'appelle le noyau d'unité. Cette terminologie est justifiée par le fait que, quel que soit $N$ un noyau relatif à $X$ et à $\xi, N U=U N=N$. On a, quels que soient $e_{1}$ et $e_{2}$ de $E, U\left(e_{1}, e_{2}\right)=\xi\left(e_{1} \cap e_{2}\right)$.

Réciproquement on dit qu'un noyau symétrique $N$ de type positif est singulier si l'on a $H(N) \cap L^{2}(\xi)=\{0\}$.

Remarque 1. Etant donné un noyau singulier $N$, on a alors, quel que soit $c>0$, 


$$
H(N+c U)=H(N) \oplus L^{2}(\xi),
$$

où $\oplus$ signifie la some directe.

Remarque 2. Soient $N$ et $N^{\prime}$ respectivement un noyau régulier satisfaisant au principe de domination et un noyau singulier. On a alors, quel que soit $p>0$,

$$
H\left(N_{p}+N^{\prime}\right)=H\left(N_{p}\right) \oplus H\left(N^{\prime}\right),
$$

où $\left(N_{p}\right)_{p>0}$ est la résolvante associée au noyau $N$.

Ceux résultent immédiatement de la proposition obtenue dans [5] ${ }^{(2)}$ et du fait que $H\left(N_{p}\right)=H(N) \cap L^{2}(\xi)$. On ne connaît pas si, dans la remarque 2, $H\left(N+N^{\prime}\right)=H(N) \oplus H\left(N^{\prime}\right)$.

Considérons finalement la contraction normale. Elle est, par définition, une transformation de la droite réelle à elle-même qui diminue la distance et conserves l'origine. On dit qu'une contraction normale $T$ opère dans un espace fonctionnel $H$ si, quelle que soit $u$ de $H, T \cdot u$ appartient à $H$ et on a $\|T \cdot u\| \leqq\|u\|$. D'après [3] et [4], on connaît bien qu'un noyau symétrique $N$ satisfait au principe de domination (resp. au principe complet du maximum) si et seulement si la contraction module (resp. toute la contraction normale) opère dans $H(N)$. La contraction module $T$ est définie par $T(a)=|a|$.

DÉFINITION 1. Soient $N_{1}$ et $N_{2}$ deux noyaux symétriques et supposons qu'il existe l'espace fonctionnel $H\left(N_{i}\right)$ au noyau $N_{i}(i=1,2)$. On dit que pour une contraction normale $T, N_{2}$ est un $T$-réduit de $N_{1}$ si, quelle que soit $u$ de $H\left(N_{1}\right) \cap H\left(N_{2}\right), T \cdot u$ appartient à $H\left(N_{1}\right) \cap H\left(N_{2}\right)$ et si l'on a

$$
\|u\|_{H\left(N_{1}\right)}^{2}-\|T \cdot u\|_{H\left(N_{1}\right)}^{2} \geqq\|u\|_{H\left(N_{2}\right)}^{2}-\|T \cdot u\|_{H\left(N_{2}\right)}^{2},
$$

où $\|\cdot\|_{H\left(N_{i}\right)}$ désigne la norme dans $H\left(N_{i}\right)$. En particulier si $T$ est la contraction module, "T-réduit" s'appelle un module-réduit. $\mathrm{Si}$, pour toute le contraction normale $T, N_{2}$ est un $T$-réduit de $N_{1}$, alors $N_{2}$ s'appelle un réduit normal de $N_{1}$.

Remarque 3. Soit $\left(N_{p}\right)_{p>0}$ une résolvante des noyaux symétriques; alors, quels que soient $p, q>0, N_{p}$ est un module-réduit de $N_{q}$. En particulier si, quel que soit $r>0, r N_{r}$ est sous-markovien, alors $N_{p}$ est un réduit normal de $N_{q}$ dès que $p \leqq q$.

(2) On a montré là que, pour deux noyaux symétriques $N_{1}$ et $N_{2}$, $H\left(N_{1}+N_{2}\right)=\left\{u_{1}+u_{2} ; u_{1} \in H\left(N_{1}\right)\right.$ et $\left.u_{2} \in H\left(N_{2}\right)\right\}$. 
On dit que $r N_{r}$ est sous-markovien si, quel que soit $e$ de $E, r N_{r}(e, X)$ $\leqq \xi(e)$. La remarque 3 est un résultat immédiat du fait que, quel que soit $p>0, N_{p}$ satisfait au principe de domination et du fait que, quelle que soit $u$ de $H\left(N_{0}\right) \cap L^{2}(\xi)$,

$$
\|u\|_{H\left(N_{p}\right)}^{2}=p \int|u(x)|^{2} d \xi(x)+\|u\|_{H\left(N_{0}\right)}^{2} .
$$

$\mathrm{Si}$, quel que soit $r>0, r N_{r}$ est sous-markovien, alors pour tout $p \geqq 0, N_{p}$ satisfait au principe complet $d u$ maximum, et donc, en utilisant aussi la présente égalité, $N_{p}$ est un réduit normal de $N_{q}$ dès que $p \leqq q$.

\section{Les deux principes du maximum}

Commençons d'abord avec le théorème suivant:

THÉORÈmE 1. Etant donné un noyau symétrique $N$ satisfaisant au principe de domination (resp. au principe complet du maximum), il existe alors une couple d'un noyau régulier $N_{0}$ satisfaisant au principe de domination (resp. au principe complet du maximum) et d'un noyau singulier $N^{\prime}$, et une seule telle que l'on ait

$$
N=N_{0}+N^{\prime} \text { et } H\left(N_{0}\right) \cap H\left(N^{\prime}\right)=\{0\} \text {. }
$$

Démonstration. Le noyau $N$ étant de type positif, il existe l'espace fonctionnel $H(N)$ au noyau $N$. On note $H_{0}$ l'adhérent de $H(N) \cap L^{2}(\xi)$ dans $H(N)$, et alors cela est un espace fonctionnel faiblement régulier. Soit $u$ une fonction de $H_{0}$; lorsque $N$ satisfait au principe de domination, on pose $v=|u|$, et lorsque $N$ satisfait au principe complet du maximum, on pose $v=\inf \left(1, u^{+}\right)$. Alors il existe une suite $\left(u_{n}\right)_{n=1}^{\infty} \operatorname{de} H(N) \cap L^{2}(\xi)$ qui converge fortement vers $u$ dans $H_{0}$ (aussi dans $H(N)$ ) avec $n \rightarrow+\infty$. On désigne par $v_{n}$ la fonction définie pour $u_{n}$ de la même manière que ci-dessus, et alors $v_{n}$ appartient à $H(N) \cap L^{2}(\xi) \subset H_{0}$. Donc

$$
\left\|v_{n}\right\|_{0}=\left\|v_{n}\right\| \leqq\left\|u_{n}\right\|=\left\|u_{n}\right\|_{0},
$$

où $\|\cdot\|_{0}$ et $\|\cdot\|$ sont respectivement les normes dans $H_{0}$ et dans $H(N)$. La suite $\left(v_{n}\right)$ étant bornée dans $H_{0}$, et donc on peut supposer qu'elle converge faiblement vers une fonction $v^{\prime}$ dans $H_{0}$ avec $n \rightarrow+\infty$. D'autre part, quels que soient $n$ un entier positif et $K$ un compact de $X$, on a

$$
\int_{K}\left|v_{n}(x)-v(x)\right| d \xi(x) \leqq \int_{K}\left|u_{n}(x)-u(x)\right| d \xi(x) \leqq A(K)\left\|u_{n}-u\right\|,
$$


où $A(K)$ est une constante dans la condition (A). Par conséquent, on a, quelle que soit $f$ de $M_{K}$,

$$
\begin{aligned}
\int v(x) f(x) d \xi(x) & =\lim _{n \rightarrow+\infty} \int v_{n}(x) f(x) d \xi(x)=\lim _{n \rightarrow+\infty}\left(u_{f}^{(0)}, v_{n}\right)_{0} \\
& =\left(u_{f}^{(0)}, v^{\prime}\right)_{0}=\int v^{\prime}(x) f(x) d \xi(x)
\end{aligned}
$$

où $u_{f}^{(0)}$ est le potentiel de $f$ dans $H_{0}$ et $(\cdot, \cdot)_{0}$ désigne le produit scalaire dans $H_{0}$, d'où $v=v^{\prime}$. On a donc

$$
\|v\|_{0} \leqq \lim _{n \rightarrow+\infty}\left\|v_{n}\right\|_{0} \leqq \lim _{n \rightarrow+\infty}\left\|u_{n}\right\|_{0}=\|u\|_{0},
$$

d'où la contraction module (resp. la contraction d'unité) opère dans $H_{0}$. Il est connu que si la contraction d'unité opère dans $H_{0}$, toutes les contractions normales opérent dans $H_{0}$. Il existe donc un noyau symétrique $N_{0}$ de type positif tel que $H_{0}=H\left(N_{0}\right)$, et $N_{0}$ est régulier.

Si $N^{\prime}=N-N_{0}$ est un noyau relatif à $X$ et à $\xi$, on a alors $H(N)$ $=H\left(N_{0}\right) \oplus H\left(N^{\prime}\right)$, d'où $N^{\prime}$ est singulier. Pour que $N^{\prime}$ soit un noyau relatif à $X$ et à $\xi$, il suffit de voir que, quelle que soit $f$ de $M_{K}^{+},\left(N f-N_{0} f\right)^{-}$ appartient à $H\left(N_{0}\right)$, parce que si c'est vrai, on a alors

$$
\left\|\left(N f-N_{0} f\right)^{-}\right\|^{2} \leqq\left(\left(N f-N_{0} f\right)^{-}, N_{0} f-N f\right)=0,
$$

qui résulte $\mathrm{du}$ fait que, quelle que soit $u$ de $H(N),\left(u^{+}, u^{-}\right) \leqq 0$, d'où $\left(N f-N_{0} f\right)^{-}=0$, et par suite,

$$
N f-N_{0} f \geqq 0 \text {. }
$$

Soit $\left(N_{p}\right)_{p>0}$ la résolvante associée au noyau $N_{0}$; alors, quels que soient $p>0$ et $q>0, N_{p} N_{q}$ a un sens, et par suite, pour toute fonction $f$ de $M_{K}^{+}, N_{p} f$ appartient à $H(N) \cap L^{2}(\xi)$. Ayant

$$
N_{p} f \geqq\left(N f-N_{p} f\right)^{-} \geqq 0,
$$

$\left(N f-N_{p} f\right)^{-}$appartient à $H\left(N_{0}\right)$. On a, quel que soit $p>0$,

$$
\left\|\left(N f-N_{p} f\right)^{-}-\left(N f-N_{0} f\right)^{-}\right\| \leqq\left\|N_{p} f-N_{0} f\right\|=\left\|N_{p} f-N_{0} f\right\|_{0} .
$$

Faisant $p \rightarrow 0$, on obtient que $\left(N f-N_{0} f\right)^{-}$appartient à $H\left(N_{0}\right)$. On arrive ainsi à la conclusion que $N^{\prime}$ est un noyau relatif à $X$ et à $\xi$. L'unicité de la forme $N=N_{0}+N^{\prime}$ est évidement déduite par la condition $H(N)=H\left(N_{0}\right) \oplus H\left(N^{\prime}\right)$. La démonstration est ainsi complète.

On ne connaît pas si l'unicité de la forme $N=N_{0}+N^{\prime}$ a lieu sans 
la condition $H\left(N_{0}\right) \cap H\left(N^{\prime}\right)=\{0\} . \quad N_{0}$ et $N^{\prime}$ s'appellent la part régulière et la part singulière de $N$, respectivement.

Exemple. Soit $X$ une surface de Riemann $\notin 0_{G}$ ou un espace de Green. Lorsque $N$ est le noyau de l'espace fonctionnel classique et muni de la norme de Dirichlet, $N_{0}$ est la fonction de Green et $N^{\prime}$ est le noyau reproduit de $H D(X)$, où $H D(X)$ est l'espace hilbertien de fonctions harmoniques dans $X$ et muni de la norme de Dirichlet.

Remarque 4. Dans le cas où $N$ est un noyau de convolution symétrique, $N^{\prime}$ satisfait toujours au principe de domination (voir [6]). Mais, dans le présent théorème, $N^{\prime}$ ne satisfait pas toujours au principe de domination. Cela se comprend en considérant l'exemple ci-dessus.

DÉFINITION 2. Soit $N$ un noyau symétrique satisfaisant au principe de domination; en posant $\tilde{N}_{p}=N_{p}+N^{\prime}$, la famille $\left(\tilde{N}_{p}\right)_{p>0}$ s'appelle la pseudo-résolvante associée au noyau $N$, où $\left(N_{p}\right)_{p>0}$ est la résolvante associée à la part régulière $N_{0}$ de $N$.

Pour une fonction $\varphi$ localement $\xi$-sommable et non-négative dans $X$, on pose

$$
H_{\varphi}=\left\{u \in H\left(N_{0}\right) ; \int|u(x)|^{2} \varphi(x) d \xi(x)<+\infty\right\} .
$$

En introduisant le produit scalaire

$$
(u, v)_{H \varphi}=(u, v)_{H\left(N_{0}\right)}+\int u(x) v(x) \varphi(x) d \xi(x)
$$

sur $H_{\varphi}, H_{\varphi}$ est un espace fonctionnel au noyau positif, et son noyau s'écrit $N_{\varphi}$. Alors $N_{\varphi}$ est régulier et satisfait au principe de domination, car la contraction module opère évidemment dans $H_{\varphi}$. Il est aussi évident que si $N$ satisfait au principe complet du maximum, $N_{\varphi}$ y satisfait aussi. On écrit $\tilde{N}_{\varphi}=N_{\varphi}+N^{\prime}$, et alors la part régulière de $\tilde{N}_{\varphi}$ est $N_{\varphi}$.

THÉORÈme 2. Soit $N$ un noyau symétrique satisfaisant au principe de domination; supposer que toutes les notations sont les mêmes que ci-dessus. Alors les cinq énoncés suivants sont équivalents:

(1) $N^{\prime}$ satisfait au principe de domination.

(2) Il existe un nombre positif $p$ tel que $N$ soit un module-réduit de $\tilde{N}_{p}$. 
(3) Quelle que soit $\varphi$ une fonction localement $\xi$-sommable et nonnégative dans $X, N$ est un module-réduit de $\tilde{N}_{\varphi}$.

(4) Quel que soit $p>0, \tilde{N}_{p}$ satisfait au principe de domination.

(5) Quelle que soit $\varphi$ une fonction localement $\xi$-sommable et nonnégative dans $X, \tilde{N}_{\varphi}$ satisfait au principe de domination.

Démonstration. Les implications (3) $\Rightarrow(2)$ et (5) $\Rightarrow$ (4) sont évidentes. Montrons d'abord l'implication (1) $\Rightarrow(5)$. Il suffit de voir que la contraction module opère dans $H\left(\tilde{N}_{\varphi}\right)$. Soient $f, g$ de $M_{K}^{+}$; alors $\left|N^{\prime} f-N^{\prime} g\right| \epsilon$ $H\left(N^{\prime}\right)$ et

$$
\left\|\mid N^{\prime} f-N^{\prime} g\right\|_{H\left(N^{\prime}\right)} \leqq\left\|N^{\prime} f-N^{\prime} g\right\|_{H\left(N^{\prime}\right)} .
$$

Soit $\left(N_{\varphi, p}\right)_{p>0}$ la résolvante associée au noyau $N_{\varphi}$; alors

$$
|| N_{\varphi, p} f+N^{\prime} f-N_{\varphi, p} g-N^{\prime} g|-| N^{\prime} f-N^{\prime} g|| \leqq\left|N_{\varphi, p} f-N_{\varphi, p} g\right| \in L^{2}(\xi) .
$$

On a donc

$$
|| N_{\varphi, p} f+N^{\prime} f-N_{\varphi, p} g-N^{\prime} g|-| N^{\prime} f-N^{\prime} g|| \in H(N) \cap L^{2}(\xi) \subset H\left(N_{0}\right),
$$

et par suite, d'après $\int\left|N_{\varphi, p} f-N_{\varphi, p} g\right|^{2} \varphi(x) d \xi(x)<+\infty$, cela appartient à $H\left(N_{\varphi}\right)$. Ayant

$$
\begin{aligned}
\| \mid N_{\varphi, p} f & +N^{\prime} f-N_{\varphi, p} g-N^{\prime} g \|_{H\left(\tilde{N}_{\varphi}\right)}^{2} \\
= & \left\|N_{\varphi, p} f+N^{\prime} f-N_{\varphi, p} g-N^{\prime} g|-| N^{\prime} f-N^{\prime} g\right\|_{H\left(\tilde{N}_{\varphi}\right)}^{2} \\
& +\left\|\mid N^{\prime} f-N^{\prime} g\right\|_{H\left(N^{\prime}\right)}^{2} \\
= & \left\|\left|N_{\varphi, p} f+N^{\prime} f-N_{\varphi, p} g-N^{\prime} g\right|-\mid N^{\prime} f-N^{\prime} g\right\|_{H\left(N_{0}\right)}^{2} \\
& \quad+\int\left(\left|N_{\varphi, p} f+N^{\prime} f-N_{\varphi, p} g-N^{\prime} g\right|-\left|N^{\prime} f-N^{\prime} g\right|\right)^{2} \varphi d \xi \\
& \quad+\left\|\left|N^{\prime} f-N^{\prime} g\right|\right\|_{H\left(N^{\prime}\right)}^{2} \\
\leqq & \left\|N_{\varphi, p} f+N^{\prime} f-N_{\varphi, p} g-N^{\prime} g\right\|_{H(N)}^{2}+\int\left|N_{\varphi, p} f-N_{\varphi, p} g\right|^{2} \varphi d \xi \\
\leqq & \left\|N_{\varphi, p} f+N^{\prime} f-N_{\varphi, p} g-N^{\prime} g\right\|_{H(N)}^{2}+\int\left|N_{\varphi, p} f-N_{\varphi, p} g\right|^{2} \varphi d \xi \\
= & \left\|N_{\varphi, p} f+N^{\prime} f-N_{\varphi, p} g-N^{\prime} g\right\|_{H\left(\tilde{N}_{\varphi}\right)}^{2} \cdot
\end{aligned}
$$

Faisant $p \rightarrow 0$, on a

$$
\left\|\left|\tilde{N}_{\varphi} f-\tilde{N}_{\varphi} g\right|\right\|_{H\left(\tilde{N}_{\varphi}\right)} \leqq\left\|\tilde{N}_{\varphi} f-\tilde{N}_{\varphi} g\right\|_{H\left(\tilde{N}_{\varphi}\right)},
$$

et donc, de la même manière que dans le théorème 1 , la contraction 
module opère dans $H\left(\tilde{N}_{\varphi}\right)$, en utilisant le fait que l'ensemble $\left\{\tilde{N}_{\varphi} f ; f \in M_{K}\right\}$ est dense dans $H\left(\tilde{N}_{\varphi}\right)$.

Montrons ensuite que (2) implique (4). Il suffit aussi de voir que, quel que soit $q>0$, la contraction module opère dans $H\left(\tilde{N}_{q}\right)$. Soient $u$ et $v$ fonctions de $H\left(N_{0}\right) \cap L^{2}(\xi)$ et de $H\left(N^{\prime}\right)$; alors $|u+v| \in H\left(\tilde{N}_{p}\right) \subset H(N)$. On désigne respectivement par $u_{1}$ et par $u_{1}^{\prime}$ la projection de $|u+v|$ sur $H\left(N_{0}\right)$ dans $H(N)$ et la projection de $|u+v|$ sur $H\left(N_{p}\right)$ dans $H\left(\tilde{N}_{p}\right)$, et on note $u_{2}=|u+v|-u_{1}$ et $u_{2}^{\prime}=|u+v|-u_{1}^{\prime}$. Alors $u_{1}-u_{1}^{\prime} \in H\left(N_{0}\right)$, $u_{2}-u_{2}^{\prime} \in H\left(N^{\prime}\right)$ et $u_{1}-u_{1}^{\prime}=u_{2}^{\prime}-u_{2}$, d'où $u_{1}=u_{1}^{\prime}$. Donc, quel que soit $q>0,|u+v|$ appartient à $H\left(\tilde{N}_{q}\right)$.

$$
\begin{aligned}
\|u+v \mid\|_{H\left(\tilde{N}_{p}\right)}^{2} & =\left\|u_{1}\right\|_{H\left(N_{p}\right)}^{2}+\left\|u_{2}\right\|_{H\left(N^{\prime}\right)}^{2} \\
& =p \int\left|u_{1}\right|^{2} d \xi+\left\|u_{1}\right\|_{H\left(N_{0}\right)}^{2}+\left\|u_{2}\right\|_{H\left(N^{\prime}\right)}^{2} \\
& =p \int\left|u_{1}\right|^{2} d \xi+\|\mid u+v\|_{H(N)}^{2} .
\end{aligned}
$$

D'après notre hypothèse, on a $\int|u|^{2} d \xi \geqq \int\left|u_{1}\right|^{2} d \xi$, d'où, quel que soit $q>0$,

$$
\begin{aligned}
\| u+ & v\left\|_{H\left(\tilde{N}_{q}\right)}^{2}-\right\| \mid u+v \|_{H\left(\tilde{N}_{q}\right)}^{2} \\
& =q \int\left(|u|^{2}-\left|u_{1}\right|^{2}\right) d \xi+\|u+v\|_{H(N)}^{2}-\|\mid u+v\|_{H(N)}^{2} \geqq 0,
\end{aligned}
$$

et par suite, on a (2) $\Rightarrow(4)$.

Supposons que l'énoncé (1) a lieu. Alors l'énoncé (3) est déduit de la même manière que ci-dessus. En effet, pour deux fonctions $u \in H\left(N_{\varphi}\right)$ et $v \in H\left(N^{\prime}\right),|u+v| \in H\left(\tilde{N}_{\varphi}\right)$ et le carré de la projection de $|u+v|$ sur $H\left(N_{\varphi}\right)$ dans $H\left(\tilde{N}_{\varphi}\right)$ est $\leqq|u|^{2}$. Donc, de la même manière que dans (2) $\Rightarrow$ (4), on arrive à la conclusion que $N$ est un module-réduit de $\tilde{N}_{\varphi}$.

Montrons finalement que l'énoncé (4) implique l'énoncé (1). On a, quelle que soit $u$ de $H\left(N^{\prime}\right),|u| \in H\left(\tilde{N}_{p}\right)$ et

$$
\|\mid u\|_{H\left(\tilde{N}_{p}\right)} \leqq\|u\|_{H\left(\tilde{N}_{p}\right)}=\|u\|_{H\left(N^{\prime}\right)} .
$$

Soit $u_{1}$ la projection de $|u|$ sur $H\left(N_{0}\right)$ dans $H(N)$; alors $u_{1}$ appartient aussi à $H\left(N_{p}\right)$ pour tout $p>0$. On a

$$
\||u|\|_{H\left(\tilde{N}_{p}\right)}^{2}=p \int\left|u_{1}\right|^{2} d \xi+\left\|u_{1}\right\|_{H\left(N_{0}\right)}^{2}+\left\||u|-u_{1}\right\|_{H\left(N^{\prime}\right)}^{2},
$$

et donc, faisant $p \rightarrow+\infty$, on obtient $u_{1}=0$, d'où $|u| \in H\left(N^{\prime}\right)$ et $\||u|\|_{H\left(N^{\prime}\right)}$ 
$\leqq\|u\|_{H\left(N^{\prime}\right)}$. Par conséquent, la contraction module opére dans $H\left(N^{\prime}\right)$, d'où (4) $\Rightarrow(1)$. La démonstration est ainsi compléte.

En considérant la présente démonstration, la remarque suivante se comprend facilement.

Remarque 5. Soit $N$ un noyau symétrique satisfaisant au principe de domination. Si la condition suivante (a) ou (b) ou (c) est vérifiée, alors $N^{\prime}$ satisfait aussi au principe de domination.

(a) $H\left(N^{\prime}\right)$ est fermé par la contraction module, c'est-à-dire, quelle que soit $u$ de $H\left(N^{\prime}\right),|u| \in H\left(N^{\prime}\right)$.

(b) Il existe une fonction $\varphi$ localement $\xi$-sommable et partout positive dans $X$ telle que $N$ soit un module-réduit de $\tilde{N}_{\varphi}$.

(c) Quelle que soit $u$ de $H(N)$, le carré de la projection de $|u|$ sur $H\left(N_{0}\right)$ dans $H(N)$ soit $\leqq|u|^{2}$.

De la même manière que dans le théorème 2 , on obtient le théorème suivant:

THÉORÈme 2'. Etant donné un noyau symétrique $N$ satisfaisant au principe complet du maximum, alors les cinq énoncés suivants sont équivalents :

(1) $N^{\prime}$ satisfait au principe complet du maximum.

(2) Il existe une fonction $\varphi$ localement $\xi$-sommable et partout positive dans $X$ telle que $N$ soit un réduit normal de $\tilde{N}_{\varphi}$.

(3) Quelle que soit $\varphi$ une fonction localement $\xi$-sommable et nonnégative dans $X, N$ est un réduit normal de $\tilde{N}_{\varphi}$.

(4) Quel que soit $p>0, \tilde{N}_{p}$ satisfait au principe complet du maximum.

(5) Quelle que soit $\varphi$ une fonction localement $\xi$-sommable et nonnégative dans $X, \tilde{N}_{\varphi}$ satisfait au principe complet du maximum.

On le montrera en utilisant la contraction d'unité en remplacement de la contraction module. On appelle la contraction d'unité la projection de la droite réelle à l'intervalle fermé $[0,1]$.

\section{Les résolvantes et les noyaux singuliers}

Enonçons d'abord une autre sorte du principe de domination.

LEMME 1. Soient $N$ un noyau symétrique satisfaisant au principe de domination et $c_{i}(i=1,2)$ une constante non-négative; supposer $c_{1} \geqq c_{2}$. Alors pour deux fonctions $f>0$ de $L^{2}(\xi)$ et $g$ de $M_{K}^{+}$, 


$$
N f+c_{1} f \leqq N g+c_{2} g \text { p.p. sur } X
$$

dès que Nf a un sens et la même inégalité a lieu presque partout pour fछ.

En effet, le noyau $N+c_{2} U$ satisfait au principe de domination (voir, par exemple, [7]), et donc il suffit de montrer l'implication suivante:

$$
N f+c f \leqq N g \text { p.p. pour } f \xi \Rightarrow N f+c f \leqq N g \text { p.p. sur } X \text {, }
$$

où $c$ est une constante non-négative. Soit $\left(K_{n}\right)_{n=1}^{\infty}$ une suite croissante de compacts de $X$ contenus dans l'ensemble $\{x \in X ; f(x)>0\}$ et telle que

$$
\int_{\cap_{n=1}^{\infty} \mathscr{E} K_{n}} f(x) d \xi(x)=0 \text {. }
$$

On a alors $N f_{n}+c f_{n} \leqq N g$ p.p. $\operatorname{sur} \operatorname{Supp}\left(f_{n}\right)$, où $f_{n}$ est la restriction de inf $(f, n)$ sur $K_{n}$. N satisfaisant au principe de domination, la présente inégalité a lieu p.p. sur $X$. Faisant $n \rightarrow+\infty$, on arrive à l'inégalité désirée.

DÉfinition 3. Soit $N$ un noyau symétrique et de type positif. Un ensemble $A$ de $E$ est dit $N$-régulier si, pour une fonction $u$ de $H(N)$, il existe une fonction $u_{A}^{\prime}$ de $H(N ; A)=\overline{\left\{u \in H(N) ; \int_{A}|u|^{2} d \xi<+\infty\right\}}$ telle que $u=u_{A}^{\prime}$ p.p. $\operatorname{sur} A$. Si, pour tout compact $K$ de $X, K$ est $N$-régulier, $N$ est dit localement régulier.

LEMME 2. Soient $N$ un noyau symétrique satisfaisant au principe de domination et $A$ un ensemble de $E$. Si la condition suivante (a) ou (b) est vérifiée, alors $A$ est $N$-régulier.

(a) Il existe une fonction $f$ de $M_{K}^{+}$telle que $N f>0$ p.p. sur $A$ et $\int_{A}|N f|^{2} d \xi<+\infty$.

(b) Quel que soit $K$ un compact de $X$ contenu dans $A, N c_{K}$ est de $L^{2}(A)$, où $c_{K}$ est la fonction caractéristique de $K$.

En effet, supposons d'abord que la condition (a) a lieu. Alors, quels que soient $n$ un entier positif et $g$ une fonction de $M_{K}^{+}, \inf (N g, n N f)$ appartient à $H(N)$ et sa norme est $\leqq\|N f\|_{H(N)}$. La suite (inf $\left.(N g, n N f)\right)$ converge donc vers une fonction $u_{A}^{\prime}$ de $H(N ; A)$ au sens de la topologie forte dans $H(N)$, d'où $N g=u^{\prime}$ p.p. sur $A$. L'ensemble $\left\{N g ; g \in M_{K}^{+}\right\}$étant dense dans $H(N)$, on arrive à la conclusion que $A$ est $N$-régulier.

Supposons ensuite que la condition (b) a lieu. On pose 


$$
A^{\prime}=\bigcup\left\{\left\{x \in A ; N c_{K}(x)>0\right\} ; K: \text { compact } \subset A\right\},
$$

et alors $A^{\prime}$ est $N$-régulier. Donc il suffit de voir que, quelle que soit $u$ de $H(N), u=0$ p.p. sur $A-A^{\prime}$. Soit $g$ une fonction de $M_{K}^{+}$; alors, quelle que soit $h$ de $M_{K}^{+}$et portée par $A-A^{\prime}$,

$$
\int N g(x) h(x) d \xi(x) \leqq\left(\int N g(x) g(x) d \xi(x)\right)^{1 / 2}\left(\int N h(x) h(x) d \xi(x)\right)^{1 / 2}=0,
$$

d'où $N g=0$ p.p. sur $A-A^{\prime}$, et par suite, quelle que soit $u$ de $H(N)$, $u=0$ p.p. sur $A-A^{\prime}$.

Pour que $A$ soit N-régulier, il suffit de supposer la condition (b') au lieu de (b).

(b') Quel que soit $K$ un compact $\subset A, N c_{K}$ appartient à $H(N ; A)$.

LEMme 3. (a) Si un noyau symétrique $N$ satisfait au principe de domination, alors il existe une suite $\left(K_{n}\right)_{n=1}^{\infty}$ de compacts de $X$ telle que $K_{n}$ soit $N$-régulier et que l'on ait $\xi\left(K_{n}\right) \leqq \xi\left(K_{n+1}\right), \xi\left(\cap \mathscr{C} K_{n}\right)=0$ et

$$
\int N c_{K_{n}}(x) c_{K_{n}}(x) d \xi(x) \leqq \int N c_{K_{n+1}}(x) c_{K_{n+1}}(x) d \xi(x)
$$

(b) Si un noyau symétrique $N$ satisfait au principe complet $d u$ maximum, il est alors localement régulier.

En effet, pour un compact $K$ de $X$ et un nombre positif $\varepsilon$ donnés, il existe un compact $K_{s}$ contenu dans $K$ tel que $\xi\left(K-K_{\varepsilon}\right)<\varepsilon$ et $N c_{K_{\sigma}}$ soit borné sur $K_{\iota}$, car $\int N c_{K}(x) c_{K}(x) d \xi(x)<+\infty$. Donc, pour une suite croissante $\left(K_{n}^{\prime}\right)$ de compacts de $X$ telle que $\bigcup_{n=1}^{\infty} K_{n}^{\prime}=X$, on peut construire facilement, par récurrence, une suite $\left(K_{n}\right)$ de compacts telle que $N c_{K_{n}}$ soit borné sur $K_{n}$ et que l'on ait $K_{n} \subset K_{n}^{\prime}, \xi\left(K_{n}\right) \leqq \xi\left(K_{n+1}\right)$, $\xi\left(\bigcap_{n=1}^{\infty} \mathscr{C} K_{n}\right)=0$ et

$$
\int N c_{K_{n}}(x) c_{K_{n}}(x) d \xi(x) \leqq \int N c_{K_{n+1}}(x) c_{K_{n+1}}(x) d \xi(x)
$$

D'après le lemme $2, K_{n}$ est $N$-régulier.

Voyons la deuxième part. Pour un compact $K$ de $X$, d'après $N(K, K)<+\infty$ et de notre hypothèse que $N$ satisfait au principe complet du maximum, il existe une suite croissante $\left(K_{n}\right)$ de compacts contenus dans $K$ telle que $N c_{K_{n}}$ soit borné sur $X$ et que l'on ait $\xi\left(K-K_{n}\right)<1 / n$. Ayant $\int\left|N c_{K_{n}}\right|^{2} d \xi<+\infty$, on obtient que $N c_{K}$ appartient à $H(N ; K)$, et 
donc, d'après la condition ci-dessus $\left(b^{\prime}\right), K$ est $N$-régulier. $K$ étant quelconque, $N$ est localement régulier.

Dans (a), on ne connaît pas si l'on peut choisir une suite croissante $\left(K_{n}\right)$ de compacts telle que $\xi\left(\cap_{n=1}^{\infty} \mathscr{C} K_{n}\right)=0$ et $K_{n}$ soit $N$-régulier.

LEMME 4. Soient $N$ un noyau symétrique satisfaisant au principe de domination et $A$ un ensemble de $E$. Alors, pour un nombre positif $p$ et pour une fonction $f$ de $M_{K}^{+}$, il existe un potentiel pur $u(f, A, p)$ dans $H(p N+U)^{(3)}$, et un seul tel que l'on ait

$$
u(f, A, p)=N f \text { p.p. sur } A, u(f, A, p) \leqq N f \text { p.p. sur } X
$$

et, quelle que soit $u$ de $H(p N+U),(u(f, A, p), u)_{(p)}=0$ dès que $u=0$ p.p. sur $A$, où $(\cdot, \cdot)_{(p)}$ est le produit scalaire dans $H(p N+U)$. Si $A$ est $N$-régulier, il existe une fonction $f_{A, p} \geqq 0$ de $L^{2}(\xi)$ et portée par $A$ telle que

$$
u(f, A, p)=p N f_{A, p}+f_{A, p}\left(=(p N+U) f_{A, p}\right) .
$$

En effet, on pose

$$
P(N ; A)=\overline{\left\{p N g+g \in H(p N+U) ; g \in M_{K}^{+}, \operatorname{Supp}(g) \subset A\right\}},
$$

où l'adhérence est au sens de la topologie forte dans $H(p N+U)$. Alors $P(N ; A)$ est un cône convexe et fermé. $N f$ appartenant à $H(p N+U)$, on peut définir la projection $u(f, A, p)$ de $N f$ sur $P(N ; A)$, et cela est évidemment un potentiel pur dans $H(p N+U)$. D'après la proposition obtenue dans [5], il existe une fonction $f_{A, p} \geqq 0$ de $L^{2}(\xi)$, et une seule telle que, quelle que soit $v$ de $L^{2}(\xi) \subset H(p N+U)$,

$$
(u(f, A, p), v)_{(p)}=\int u(x) f_{A, p}(x) d \xi(x) .
$$

Evidemment, quelle que soit $u$ de $H(p N+U),(u(f, A, p), u)_{(p)}=0$ dès que $u=0$ p.p. sur $A$, et donc, $f_{A, p}$ est portée par $A$. On désigne par $f_{A, p}^{(n)}$ la restriction de $\inf \left(f_{A, p}, n\right)$ sur $K_{n}$, où $\left(K_{n}\right)$ est une suite croissante de compacts contenus dans $A$ telle que $\xi\left(A-\cup_{n=1}^{\infty} K_{n}\right)=0$. Alors, quelle que soit $u$ de $H(p N+U)$ avec $u \geqq 0$,

$$
(u(f, A, p), u)_{(p)} \geqq\left(p N f_{A, p}^{(n)}+f_{A, p}^{(n)}, u\right)_{(p)},
$$

d'où $u(f, A, p) \geqq p N f_{A, p}^{(n)}+f_{A, p}^{(n)}$. Faisant $n \rightarrow+\infty$, on obtient que $p N f_{A, p}$

(3) Soit $H$ un espace fonctionnel; un élement $u$ de $H$ s'appelle un potentiel pur dans $H$ si, quelle que soit $v$ de $H,(u, v) \geqq 0$ dès que $v \geqq 0$ (voir [3]). 
$+f_{A, p}$ a un sens et $u(f, A, p) \geqq p N f_{A, p}+f_{A, p}$. Donc, quelle que soit $v$ de $H(p N+U)$,

$$
(u(f, A, p), v)_{(p)}=\int v(x) f_{A, p}(x) d \xi(x)=\left(p N f_{A, p}+f_{A, p}, v\right)_{(p)}
$$

dès que $\int_{A}|v|^{2} d \xi<+\infty$, et par suite, quelle que soit $u$ de $H(p N+U ; A)$,

$$
(u(f, A, p), u)_{(p)}=\left(p N f_{A, p}+f_{A, p}, u\right)_{(p)} .
$$

Si $A$ est $N$-régulier, alors, pour toute $u$ de $H(p N+U)$, il existe une fonction $u_{A, p}^{\prime}$ de $H(p N+U)$ telle que $u=u_{A, p}^{\prime}$ p.p. sur $A$, car $A$ est aussi $(p N+U)$-régulier. Donc

$$
\begin{aligned}
(u(f, A, p), u)_{(p)} & =\left(u(f, A, p), u_{A, p}^{\prime}\right)_{(p)} \\
& =\left(p N f_{A, p}+f_{A, p}, u_{A, p}^{\prime}\right)_{(p)}=\left(p N f_{A, p}+f_{A, p}, u\right)_{(p)},
\end{aligned}
$$

d'où $u(f, A, p)=p N f_{A, p}+f_{A, p}$. D'après le fait que $u(f, A, p)$ est la projection de $N f$ sur $P(N ; A)$, on a

$p N f_{A, p}+f_{A, p} \geqq N f$ p.p. sur $A$ et $p N f_{A, p}+f_{A, p}=N f$ p.p. pour $f_{A, p} \xi$, et par suite, d'après le lemme 1 ,

$$
p N f_{A, p}+f_{A, p} \leqq N f \text { p.p. sur } X \text { et } p N f_{A, p}+f_{A, p}=N f \text { p.p. } \operatorname{sur} A .
$$

Supposons que $A$ n'est pas $N$-régulier. Evidemment, pour une suite croissante $\left(K_{n}\right)$ de compacts de $X$ telle que $A \supset K_{n}$ et $\xi\left(A-\bigcup_{n=1}^{\infty} K_{n}\right)$ $=0$, la suite $\left(u\left(f, K_{n}, p\right)\right)_{n=1}^{\infty}$ converge fortement vers $u(f, A, p)$ dans $H(p N+U)$ avec $n \rightarrow+\infty$. D'autre part, pour un compact $K$ de $X$, on peut choisir une suite $\left(K_{n}^{\prime}\right)$ de compacts contenus dans $K$ telle que $\xi\left(K-K_{n}^{\prime}\right)<1 / n$ et $K_{n}^{\prime}$ soit $N$-régulier. On obtient alors que la suite $\left(u\left(f, K_{n}^{\prime}, p\right)\right)$ est bornée dans $H(p N+U)$, et donc on peut supposer qu'elle converge faiblement vers une fonction $u$ de $H(p N+U)$ dans $H(p N+U)$ avec $n \rightarrow+\infty$. $u$ est évidemment un potentiel pur dans $H(p N+U)$ et, quelle que soit $v$ de $H(p N+U),(u, v)_{(p)}=0$ dès que $v=0$ p.p. sur $A$. On a

$$
\begin{aligned}
& \varlimsup_{n, m \rightarrow+\infty} \int_{K}\left|u\left(f, K_{n}^{\prime}, p\right)-u\left(f, K_{m}^{\prime}, p\right)\right| d \xi \\
& \quad \leqq \lim _{n, m \rightarrow+\infty} \int_{\left(K-K_{n}^{\prime}\right) \cup\left(K-K_{m}^{\prime}\right)} N f(x) d \xi(x)=0,
\end{aligned}
$$

d'où $u=N f$ p.p. sur $K$, et par suite $u=u(f, K, p)$. D'après l'inégalité 


$$
\lim _{n \rightarrow+\infty}\left\|u\left(f, K_{n}^{\prime}, p\right)\right\|_{(p)} \leqq\|u(f, K, p)\|_{(p)},
$$

la suite $\left(u\left(f, K_{n}^{\prime}, p\right)\right)$ converge fortement vers $u(f, K, p)$ dans $H(p N+U)$ avec $n \rightarrow+\infty$. Par conséquent, pour notre ensemble $A$, il existe une suite $\left(K_{n}^{\prime \prime}\right)$ de compacts contenus dans $A$ telle que $\xi\left(A-\bigcup_{n=1}^{\infty} K_{n}^{\prime \prime}\right)=0$,

$$
\lim _{n \rightarrow+\infty}\left\|u\left(f, K_{n}^{\prime \prime}, p\right)-u(f, A, p)\right\|_{(p)}=0
$$

et $K_{n}^{\prime \prime}$ soit $N$-régulier, d'où

$$
u(f, A, p) \leqq N f \text { p.p. sur } X \text { et } u(f, A, p)=N f \text { p.p. sur } A .
$$

Pour un ensemble $A$ de $E, p N f_{A, p}+f_{A, p}$ s'appelle le potentiel balayé de $N f$ sur $A$ relativement au noyau $p N+U$. Lorsque $A$ est $N$-régulier, cela est au sens usuel.

Proposition 1. Soient $N$ un noyau symétrique satisfaisant au principe de domination et $A$ un ensemble de $E$. En posant, pour deux ensembles $e_{1}, e_{2}$ de $E_{0}$,

$$
N_{A, p}\left(e_{1}, e_{2}\right)=\int_{A \cap e_{1}}\left(c_{e_{2}}\right)_{A, p}(x) d \xi(x),
$$

$N_{A, p}$ est un noyau symétrique et portée par $A \times A$ et la famille $\left(N_{A, p}\right)_{p>0}$ est une résolvante. Il existe une suite $\left(K_{n}\right)_{n=1}^{\infty}$ de compacts de $X$ telle que l'on ait $\xi\left(X-\cup_{n=1}^{\infty} K_{n}\right)=0$ et que, quels que soient $p>0$ et $f$ de $M_{K}, N_{0} N_{n, p} f$ converge fortement vers $N_{0} N_{p} f$ et $p N^{\prime} N_{n, p} f$ converge fortement vers $N^{\prime} f$ dans $H(N)$ avec $n \rightarrow+\infty$, où $N_{n, p}=N_{K_{n}, p}$ et $N_{0},\left(N_{p}\right)_{p>0}$ et $N^{\prime}$ sont respectivement la part régulière de $N$ et la résolvante associée au noyau $N_{0}$ et la part singulière de $N$.

On préparera d'abord un lemme.

LEMme 5. Etant donné un noyau symétrique $N$ satisfaisant au principe de domination, alors, quel que soit c une constante positive, la part régulière de $N+c U$ est égale à $N_{0}+c U$, où $N_{0}$ est la part régulière de $N$.

En effet, $N_{0}+c U$ est régulier, car, pour une fonction quelconque $u$ de $H\left(N_{0}+c U\right)$, on peut écrire $u=u_{1}+u_{2}$, où $u_{1} \in H\left(N_{0}\right)$ et $u_{2} \in L^{2}(\xi)$, et on a

$$
\|u\|_{H\left(N_{0}+c U\right)} \leqq\left(\left\|u_{1}\right\|_{H\left(N_{0}\right)}^{2}+\int\left|u_{2}(x)\right|^{2} d \xi(x)\right)^{1 / 2}
$$


D'après $N+c U=\left(N_{0}+c U\right)+N^{\prime}$, il suffit de voir $H\left(N_{0}+c U\right) \cap H\left(N^{\prime}\right)$ $=\{0\}$, où $N^{\prime}$ est la part singulière de $N$. Soit $u$ une fonction de $H\left(N_{0}+c U\right) \cap H\left(N^{\prime}\right)$; alors elle s'exprime sous la forme $u=u_{1}+u_{2}$, où $u_{1} \in H\left(N_{0}\right) \subset H(N)$ et $u_{2} \in L^{2}(\xi)$, et donc, d'après $u \in H\left(N^{\prime}\right) \subset H(N), u_{2}$ appartient à $L^{2}(\xi) \cap H(N) \subset H\left(N_{0}\right)$. Par conséquent, $u \in H\left(N_{0}\right)$, d'où $u=0$.

Démonstration de la proposition 1. Il est évident que $N_{A, p}$ est un noyau relatif à $X$ et à $\xi$ et portée par $A \times A$. Si $A$ est $N$-régulier, alors, quels que soient $e_{1}, e_{2}$ de $E$ avec $e_{1} \cup e_{2} \subset A$,

$$
\begin{aligned}
& N\left(e_{1}, e_{2}\right)-N_{A, p}\left(e_{1}, e_{2}\right)=p N N_{A, p}\left(e_{1}, e_{2}\right) \\
& =p \int N c_{e_{1}}(x)\left(c_{e_{2}}\right)_{A, p}(x) d \xi(x)=p \int\left(p N\left(c_{e_{1}}\right)_{A, p}+\left(c_{e_{1}}\right)_{A, p}\right)\left(c_{e_{2}}\right)_{A, p} d \xi \\
& =p \int\left(p N\left(c_{e_{2}}\right)_{A, p}+\left(c_{e_{2}}\right)_{A, p}\right)\left(c_{e_{1}}\right)_{A, p} d \xi=p \int N c_{e_{2}}(x)\left(c_{e_{1}}\right)_{A, p}(x) d \xi(x) \\
& =p N N_{A, p}\left(e_{2}, e_{1}\right)
\end{aligned}
$$

d'où $N_{A, p}$ est symétrique. Ayant $p N N_{A, p}=N-N_{A, p}$ sur $E_{A} \times E_{A}$, où $E_{A}=\{e \in E ; e \subset A\}$, la famille $\left(N_{A, p}\right)_{p>0}$ résulte une résolvante de la manière usuelle (cf. par exemple, [7]).

Supposons, en général, que $A$ n'est pas $N$-régulier ; alors, de la même manière que dans le lemme 4 , il existe une suite $\left(K_{n}\right)$ de compacts contenus dans $A$ telle que, quels que soient $p>0$ et $f$ de $M_{K}$, la suite $\left(u\left(f, K_{n}, p\right)\right)$ converge fortement vers $u(f, A, p)$ dans $H(p N+U)$ avec $n \rightarrow+\infty$ et que $K_{n}$ soit $N$-régulier. On a alors, pour toute $f$,

$$
\int\left|N_{K_{n}, p} f-N_{A, p} f\right|^{2} d \xi \leqq\left\|u\left(f, K_{n}, p\right)-u(f, A, p)\right\|_{(p)}^{2},
$$

et donc, la famille $\left(N_{A, p}\right)_{p>0}$ est une résolvante. Soit $A=X$; alors $N_{A, p}$ $=N_{p}$, car $H(N ; X)=H\left(N_{0}\right)$, et donc, il existe une suite $\left(K_{n}\right)$ de compacts de $X$ telle que $K_{n}$ soit $N$-régulier et que, quels que soient $p>0$ et $f$ de $M_{K}$, la suite $\left(p N N_{n, p} f+N_{n, p} f\right)_{n=1}^{\infty}$ converge fortement vers $N f$ dans $H(p N+U)$ avec $n \rightarrow+\infty$. On a évidemment $\xi\left(\cap_{n=1}^{\infty} \mathscr{C} K_{n}\right)=0$. D'après le lemme 5 , on a

$$
\begin{aligned}
& \left\|p N N_{n, p} f+N_{n, p} f-N f\right\|_{(p)}^{2} \\
& =\left\|p N_{0} N_{n, p} f+N_{n, p} f-N_{0} f\right\|_{(p)}^{2}+\left\|p N^{\prime} N_{n, p} f-N^{\prime} f\right\|_{(p)}^{2} \\
& =\left\|p N_{0} N_{n, p} f-p N_{0} N_{p} f\right\|_{H\left(N_{0}\right)}^{2}+\int\left|N_{n, p} f-N_{p} f\right|^{2} d \xi \\
& \quad+\left\|p N^{\prime} N_{p, n} f-N^{\prime} f\right\|_{H\left(N^{\prime}\right)},
\end{aligned}
$$


et donc, les suites $\left(N_{n, p} f\right),\left(N_{0} N_{n, p} f\right)$ et $\left(p N^{\prime} N_{p, n} f\right)$ convergent fortement vers $N_{0} f, N_{0} N_{p} f$ et $N^{\prime} f$ dans $L^{2}(\xi)$, dans $H\left(N_{0}\right)$ et dans $H\left(N^{\prime}\right)$ avec $n \rightarrow+\infty$, respectivement.

On dit que $\left(N_{A, p}\right)_{p>0}$ la résolvante sur $A$ associée au noyau $N$. On obtient facilement que si $A \subset B$ et ils sont $N$-réguliers, on a alors $N_{A, p}$ $\geqq N_{B, p}$ sur $A \times A$.

Remarque 6. Soient $N$ un noyau symétrique satisfaisant au principe de domination et $\left(N_{p}\right)_{p>0}$ la résolvante associée à la part régulière $N_{0}$ de $N$; alors, quel que soit $p>0, N^{\prime} N_{p}$ a un sens et on a $N^{\prime} \geqq p N^{\prime} N_{p}$, où $N^{\prime}$ est la part singulière de $N$.

On choit une suite $\left(K_{n}\right)_{n=1}^{\infty}$ des compacts de $X$ telle que, quels que soient $p>0$ et $f$ de $M_{K}$, les suites $\left(N_{n, p} f\right)_{n=1}^{\infty}$ et $\left(p N^{\prime} N_{n, p} f\right)_{n=1}^{\infty}$ convergent fortement vers $N_{p} f$ et $N^{\prime} f$ dans $L^{2}(\xi)$ et dans $H\left(N^{\prime}\right)$ avec $n \rightarrow+\infty$, respectivement, où $\left(N_{n, p}\right)_{p>0}$ est la résolvante sur $K_{n}$ associée au noyau $N$. On a alors, quelles que soient $f$ et $g$ de $M_{K}^{+}$,

$$
\begin{aligned}
\int N^{\prime} f(x) g(x) d \xi(x) & =\lim _{n \rightarrow+\infty} p \int N^{\prime} N_{n, p} f(x) g(x) d \xi(x) \\
& =\lim _{n \rightarrow+\infty} p \int N^{\prime} g(x) N_{n, p} f(x) d \xi(x) \geqq p \int N^{\prime} g(x) N_{p} f(x) d \xi(x)
\end{aligned}
$$

d'où $N^{\prime} N_{p}$ a un sens et $N^{\prime} \geqq p N^{\prime} N_{p}$.

LeMme 6. Si un noyau symétrique $N$ satisfait au principe complet $d u$ maximum, alors, quels que soient $A$ de $E_{0}$ et $p>0, p N_{A, p}$ est sousmarkovien. Pour e de $E_{0}, p N_{A, p}(e, X)$ est croissante avec $A$.

En effet, pour une fonction $f$ de $M_{K}^{+}$et pour deux ensembles $A, B$ de $E_{0}$ avec $A \subset B$,

$$
p N\left(N_{A, p} f\right)+N_{A, p} f \leqq p N\left(N_{B, p} f\right)+N_{B, p} f \leqq N f
$$

p.p. sur $X$. D'après le lemme suivant et le principe complet du maximum pour $p N+U$,

$$
p \int N_{A, p} f d \xi \leqq p \int N_{B, p} f d \xi \leqq \int f d \xi,
$$

d'où notre lemme.

LEMME 7. Si un noyau symétrique $N$ satisfait au principe complet 
du maximum, alors, quelles que soient $f, g$ de $M_{K}^{+}, \int f d \xi \leqq \int g d \xi$ dès que $N f \leqq N g$ p.p. sur $\operatorname{Supp}(f)$.

Voir [7].

DÉfinition 4. Soit $N_{0}$ un noyau régulier; on note $D_{s}\left(N_{0}\right)$ (resp. $C_{s}\left(N_{0}\right)$ ) la totalité des noyaux singuliers $N^{\prime}$ tels que $H\left(N_{0}\right) \cap H\left(N^{\prime}\right)=\{0\}$ et $N_{0}+N^{\prime}$ satisfasse au principe de domination (resp. au principe complet du maximum).

D'après le théorème 1 , pour qu'un noyau régulier $N_{0}$ satisfasse au principe de domination (resp. au principe complet du maximum), il faut et il suffit que $D_{s}\left(N_{0}\right) \neq \emptyset$ (resp. $C_{s}\left(N_{0}\right) \neq \emptyset$ ). On obtient aussi, d'après la remarque 6, que si, pour un noyau régulier $N_{0}, D_{s}\left(N_{0}\right) \ni 1$, alors $N_{0}$ satisfait au principe complet du maximum, car, quel que soit $p>0, p N_{p}$ est sous-markovien, où $\left(N_{p}\right)_{p>0}$ est la résolvante associée au noyau $N_{0}$.

Proposition 2. Soit $N_{0}$ un noyau régulier; alors, pour que $C_{s}\left(N_{0}\right) \neq \emptyset$ et $C_{s}\left(N_{0}\right) \neq\{0\}$, il faut et il suffit que $N_{0}$ satisfasse au principe complet du maximum et $H\left(N_{0}+U\right) \nexists 1$. Dans ce cas, $C_{s}\left(N_{0}\right)$ contient toutes les constantes non-négatives.

En effet, si $C_{s}\left(N_{0}\right) \neq \emptyset$ et $C_{s}\left(N_{0}\right) \neq\{0\}$, alors, quel que soit $c>0$, $N_{0}+c U$ satisfait au principe complet du maximum, et $C_{s}\left(N_{0}\right)$ $\subset C_{s}\left(N_{0}+c U\right)$. Si $H\left(N_{0}+U\right) \ni 1$, toute la constante positive appartient à $H\left(N_{0}+U\right)$ et elle est un potentiel pur dans $H\left(N_{0}+U\right)$. Soit $N^{\prime} \neq 0$ de $C_{s}\left(N_{0}+U\right)$; alors, quels que soient $a>0$ et $f$ de $M_{K}^{+}$, inf $\left(N_{0} f+N^{\prime} f\right.$ $+f, a)$ appartient à $H\left(N_{0}+U\right)$ et cela est un potentiel pur dans $H\left(N_{0}+U\right)$. Faisant $a \rightarrow+\infty$, on a $N^{\prime} f \in H\left(N_{0}+U\right)$, d'où une contradiction.

Si $N_{0}$ satisfait au principe complet du maximum et $H\left(N_{0}+U\right) \nexists 1$, on obtient que $C_{s}\left(N_{0}\right)$ contient toutes les constantes non-négatives. Soit $a$ une constante positive; supposer que, pour deux fonctions $f, g$ de $M_{K}^{+}$,

$$
a \int f d \xi+N_{0} f \leqq a \int g d \xi+N_{0} g+1 \text { p.p. } \operatorname{sur} \operatorname{Supp}(f)
$$

Si $a \int f d \xi \leqq a \int g d \xi+1$, alors, d'après le principe complet du maximum pour $N_{0}$, la même inégalité a lieu p.p. sur $X$. Supposons $a \int f d \xi>a \int g d \xi$ 
+1 ; alors $N_{0} f<N_{0} g$ p.p. $\operatorname{sur} \operatorname{Supp}(f)$. D'après le lemme $7, \int f d \xi \leqq$ $\int g d \xi$, d'où une contradiction.

DÉFINITION 5. Soit $N_{0}$ un noyau symétrique; un noyau symétrique $N$ est dit $N_{0}$-invariant si l'on a

$$
N_{0} N=N N_{0}=N \text {. }
$$

THÉORÈme 3. Soient $N_{0}$ un noyau régulier satisfaisant au principe complet du maximum et $\left(N_{p}\right)_{p>0}$ la résolvante associée au noyau $N_{0}$; supposer ensuite $C_{s}\left(N_{0}\right) \neq\{0\}$. Alors les trois énoncés suivants sont équivalents :

(1) Quels que soient $N^{\prime}$ de $C_{s}\left(N_{0}\right)$ et $p>0, N^{\prime}$ est $\left(p N_{p}\right)$-invariant.

(2) Quels que soient $N^{\prime}$ de $C_{s}\left(N_{0}\right), K$ un compact de $X$ et $p>0$, on a

$$
N_{K, p}(e, X) \leqq N_{p}(e, X)
$$

pour tout $e$ de $E$, où $\left(N_{K, p}\right)_{p>0}$ est la résolvante sur $K$ associée au noyau $N_{0}+N^{\prime}$.

(3) Quel que soit $p>0, p N_{p}$ est markovien, c'est-à-dire, quel que soit $e$ de $E, p N_{p}(e, X)=\xi(e)$.

Démonstration. Supposons d'abord l'énoncé (1) ; alors, d'après $C_{s}\left(N_{0}\right)$ $\ni 1, p N_{p}$ est markovien, d'où $N_{K, p}(e, X) \leqq N_{p}(e, X)$ pour tout $e$ de $E$, car, d'après le lemme $6, p N_{K, p}$ est sous-markovien, d'où (1) $\Rightarrow(2)$.

Montrons ensuite l'implication (2) $\Rightarrow(3)$. Soient $\left(K_{n}\right)_{n=1}^{\infty}$ une suite croissante de compacts de $X$ avec $\bigcup_{n=1}^{\infty} K_{n}=X$ et $\left(N_{n, p}\right)_{p>0}$ la résolvante sur $K_{n}$ associée au noyau $N+1$. Alors, d'après la proposition 1 ,

$$
\lim _{n \rightarrow+\infty} p N_{n, p}(e, X)=\xi(e)
$$

pour tout $e$ de $E_{0}$, d'où $p N_{p}$ est markovien.

On supposera finalement l'énoncé (3). Soit $N^{\prime}$ un noyau singulier de $C_{s}\left(N_{0}\right)$; alors $N^{\prime}+1$ appartient aussi à $C_{s}\left(N_{0}\right)$. On pose $N=N_{0}+N^{\prime}$ +1 , et on prend une suite croissante $\left(K_{n}\right)_{n=1}^{\infty}$ de compacts de $X$ avec $\bigcup_{n=1}^{\infty} K_{n}=X . \quad\left(N_{n, p}\right)_{p>0}$ désigne la résolvante sur $K_{n}$ associée au noyau $N$. Ayant $1 \in H\left(N^{\prime}+1\right)$, on obtient, quel que soit $e$ de $E_{0}$,

$$
\lim _{n \rightarrow+\infty} p N_{n, p}(e, X)=\xi(e)=p N_{p}(e, X) .
$$

On a, d'autre part, 


$$
\lim _{n \rightarrow+\infty} p N_{n, p}\left(e_{1}, e_{2}\right)=\dot{p} N_{p}\left(e_{1}, e_{2}\right) \text { sur } E_{0} \times E_{0} .
$$

Par conséquent, pour un nombre $0<\varepsilon<1$ donné et pour un ensemble $e_{0}$ de $E_{0}$, il existe un compact $K_{\varepsilon}$ de $X$ tel que, pour tout entier $n$ suffisamment grand,

$$
p N_{p, n}\left(e_{0}, C K_{\varepsilon}\right)<\varepsilon \xi\left(e_{0}\right) .
$$

Soit $f$ une fonction de $M_{K}^{+}$telle que $N^{\prime} f$ soit borné sur $X$; on a alors

$$
\begin{aligned}
\int_{e_{0}} N^{\prime} f d \xi & =\lim _{n \rightarrow+\infty} p \int N^{\prime} f(x) N_{n, p} c_{e_{0}}(x) d \xi(x) \\
& \leqq \lim _{n \rightarrow+\infty} p \int_{K_{\varepsilon}} N^{\prime} f(x) N_{n, p} c_{e_{0}}(x) d \xi(x)+\varepsilon \xi\left(e_{0}\right)\left(\underset{x \in X}{\operatorname{ess} . \sup } N^{\prime} f(x)\right) \\
& \leqq p \int N^{\prime} f(x) N_{p} c_{e_{0}}(x) d \xi(x)+\varepsilon\left(\underset{x \in X}{\operatorname{ess} . \sup } N^{\prime} f(x)\right) \xi\left(e_{0}\right),
\end{aligned}
$$

où $c_{e_{0}}$ est la fonction caratéristique de $e_{0}$. Faisant $\varepsilon \rightarrow 0$, on a

$$
\int_{e_{0}} N^{\prime} f d \xi \leqq p \int N^{\prime} f(x) N_{p} c_{e_{0}}(x) d \xi(x) .
$$

Donc $N^{\prime} \leqq p N^{\prime} N_{p}$, car, d'après le principe complet du maximum pour $N$, pour une fonction $f$ de $M_{K}^{+}$, il existe une suite croissante $\left(K_{n}\right)_{n=1}^{\infty}$ de compacts de $X$ telle que

$$
\int_{C K_{n}} f d \xi<\frac{1}{n}
$$

et $N f_{n}$ soit borné sur $X$, où $f_{n}$ est la restriction de $f$ sur $K_{n}$. En combinant avec la remarque 6 , on obtient que $N^{\prime}$ est $\left(p N_{p}\right)$-invariant. La démonstration est ainsi complète.

Remarque 7. Soient $N_{0}$ un noyau régulier satisfaisant au principe de domination, $\left(N_{p}\right)_{p>0}$ la résolvante associée au noyau $N_{0}$ et $N^{\prime}$ un noyau singulier; on suppose qu'il existe un nombre positif $p_{0}$ tel que $p_{0} N_{p_{0}}$ soit markovien. Si $N^{\prime}$ est $\left(p_{0} N_{p_{0}}\right)$-invariant, on a alors $H\left(N_{0}\right) \cap H\left(N^{\prime}\right)=\{0\}$.

D'après $H\left(N_{0}\right) \subset H\left(N_{0}+\left(1 / p_{0}\right) U\right)$, il suffit de voir que $H\left(N_{0}+\left(1 / p_{0}\right) U\right)$ $\cap H\left(N^{\prime}\right)=\{0\}$. Ayant

$$
N_{0}+\frac{1}{p_{0}} U=\frac{1}{p_{0}} \sum_{n=0}^{\infty}\left(p_{0} N_{p_{0}}\right)^{n},
$$

où $(\cdot)^{0}=U$ et $(\cdot)^{n}=(\cdot)^{n-1}(\cdot)(n \geqq 2)$, et $p_{0} N_{p_{0}}$ étant markovien, on obtient que, quelles que soient $u, v$ de $H\left(N_{0}+\left(1 / p_{0}\right) U\right)$, 


$$
\begin{aligned}
(u, v)_{H\left(N_{0}+\left(1 / p_{0}\right) U\right)} & =\frac{1}{2} p_{0} \iint(u(x)-u(y))(v(x)-v(y)) p_{0} N_{p_{0}}(d x, d y) \\
& =p_{0} \iint u(x)(v(x)-v(y)) p_{0} N_{p_{0}}(d x, d y) .
\end{aligned}
$$

D'autre part, l'ensemble $\left\{N^{\prime} f \in H\left(N^{\prime}\right) ; f \in M_{K}\right\}$ étant dense dans $H\left(N^{\prime}\right)$, on a, quelle que soit $v$ de $H\left(N^{\prime}\right), v=p_{0} N_{p_{0}} v$.

Soit $u$ une fonction quelconque de $H\left(N_{0}+\left(1 / p_{0}\right) U\right) \cap H\left(N^{\prime}\right)$; alors, quelle que soit $f$ de $M_{K}$, on a

$$
\begin{aligned}
(u, f)_{H\left(N_{0}+\left(1 / p_{0}\right) U\right)} & =p_{0} \iint f(x)(u(x)-u(y)) p_{0} N_{p_{0}}(d x, d y) \\
& =p_{0} \int f(x)\left(u(x)-p_{0} N_{p_{0}} u(x)\right) d \xi(x)=0 .
\end{aligned}
$$

$M_{K}$ est dense dans $H\left(N_{0}+\left(1 / p_{0}\right) U\right)$, car, d'après la proposition obtenue dans [5], pour une fonction $v$ de $H\left(N_{0}+\left(1 / p_{0}\right) U\right)$, il existe une fonction $g$ de $L^{2}(\xi)$, et une seule telle que, quelle que soit $w$ de $L^{2}(\xi) \subset$ $H\left(N_{0}+\left(1 / p_{0}\right) U\right)$,

$$
(v, w)_{H\left(N_{0}+\left(1 / p_{0}\right) U\right)}=\int w g d \xi .
$$

Par conséquent $u=0$, d'où $H\left(N_{0}+\left(1 / p_{0}\right) U\right) \cap H\left(N^{\prime}\right)=\{0\}$.

On note $L_{\mathrm{loc}}^{+}(\xi)$ la totalité des fonctions non-négatives et localement $\xi$-sommables dans $X$. On obtient, d'autre part, la proposition suivante:

Proposition 3. Soit $N_{0}$ un noyau régulier satisfaisant au principe complet du maximum; on suppose que, quel que soit $p>0, p N_{p}$ est markovien, où $\left(N_{p}\right)_{p>0}$ est la résolvante associée au noyau $N_{0}$. Pour une fonction $\varphi$ de $L_{\mathrm{loc}}^{+}(\xi), N_{\varphi}$ est le noyau régulier défini dans la définition 2. Alors, pour un noyau singulier $N^{\prime}$ de $C_{s}\left(N_{0}\right)$, on a, quels que soient $p>0$ et $f$ de $M_{K}$,

$$
N^{\prime} f=p N^{\prime} N_{\varphi, p} f+N^{\prime}\left(\left(N_{\varphi, p} f\right) \varphi\right),
$$

où $\left(N_{\varphi, p}\right)_{p>0}$ est la résolvante associée au noyau $N_{\varphi}$.

Cela est un résultat immédiat du lemme suivant:

LEMME 8. Soient $N_{0}$ un noyau régulier satisfaisant au principe de domination et une fonction de $L_{1 \mathrm{oc}}^{+}(\xi) ;\left(N_{p}\right)_{p>0}$ et $\left(N_{\varphi, p}\right)_{p>0}$ désignent respectivement les résolvantes associées au noyau $N_{0}$ et au noyau $N_{\varphi}$. Alors on a, quelle que soit $f$ de $M_{K}$, 


$$
N_{p} f=N_{\varphi, p} f+N_{p}\left(\left(N_{\varphi, p} f\right) \varphi\right) .
$$

En effet, on a, quelles que soient $u \operatorname{de} H\left(N_{\varphi, p}\right)$ et $f \operatorname{de} M_{K}$,

$$
\begin{aligned}
\left(N_{\varphi, p} f, u\right)_{H\left(N_{\varphi}, p\right)} & =\int u(x) f(x) d \xi(x) \\
& =\int u(x) N_{\varphi, p} f(x) \varphi(x) d \xi(x)+\left(N_{\varphi, p} f, u\right)_{H\left(N_{p}\right)} .
\end{aligned}
$$

On suppose d'abord que $\varphi$ est bornée sur $X$, et alors on a $H\left(N_{\varphi, p}\right)=H\left(N_{p}\right)$. Donc, quelle que soit $u$ de $H\left(N_{p}\right)$,

$$
\int\left(\left(N_{\varphi, p} f\right) \varphi-f\right) u d \xi+\left(N_{\varphi, p} f, u\right)_{H\left(N_{p}\right)}=0,
$$

et par suite, $N_{p}\left(\left(N_{\varphi, p} f\right) \varphi-f\right)$ a un sens et appartient à $H\left(N_{p}\right)$, d'où

$$
N_{p} f=N_{\varphi, p} f+N_{p}\left(\left(N_{\varphi, p} f\right) \varphi\right) .
$$

On a, en même temps, $N_{p} \geqq N_{\varphi, p}$. En général, poser $\varphi_{n}=\inf (\varphi, n)$; alors la suite $\left(N_{\varphi_{n, p}}\right)_{n=1}^{\infty}$ est décroissante pour tout $p>0$. Si $n<m$, $N_{\varphi_{n, p}}-N_{\varphi_{m, p}}$ est un noyau symétrique et de type positif, et donc, $N_{\varphi_{n, p}}$ $-N_{\varphi, p}^{\prime}$ est aussi un noyau symétrique et de type positif, où $N_{\varphi, p}^{\prime}=$ $\lim _{n \rightarrow+\infty} N_{\varphi_{n, p}}$. On a, pour une fonction $u \operatorname{de~} H\left(N_{\varphi, p}^{\prime}\right)$,

$$
\begin{aligned}
\|u\|_{H\left(N_{\varphi}^{\prime}, p\right)}^{2} & \geqq \lim _{n \rightarrow+\infty}\|u\|_{H\left(N_{\left.\varphi_{n}, p\right)}\right.}^{2}=\lim _{n \rightarrow+\infty} \int|u(x)|^{2} \varphi_{n}(x) d \xi(x)+\|u\|_{H\left(N_{p}\right)}^{2} \\
& =\int|u(x)|^{2} \varphi(x) d \xi(x)+\|u\|_{H\left(N_{p}\right)}^{2}=\|u\|_{H\left(N_{\varphi, p)}\right.}^{2},
\end{aligned}
$$

et par suite, on a $H\left(N_{\varphi, p}^{\prime}\right) \subset H\left(N_{\varphi, p}\right)$ et $N_{\varphi, p}-N_{\varphi, p}^{\prime}$ est de type positif. D'autre part, quel que soit $n$ un entier positif, $N_{\varphi_{n, p}}-N_{\varphi, p}$ étant de type positif, $N_{\varphi, p}^{\prime}-N_{\varphi, p}$ est aussi de type positif, d'où $N_{\varphi, p}=N_{\varphi, p}^{\prime}$. D'après le théorème de Lebesgue, on a

$$
N_{p} f=N_{\varphi, p} f+N_{p}\left(\left(N_{\varphi, p} f\right) \varphi\right) .
$$

Remarque 8. D'après la présente proposition, on a, en général, $C_{s}\left(N_{0}\right) \not \supset C_{s}\left(N_{\varphi}\right)$. Cela se comprend en considérant une surface de Riemann $X$ et une fonction non-négative $\varphi(\neq 0)$ telles que $\Phi H D(X)-L^{2} \neq \emptyset$, où $\Phi H D(X)$ est l'espace des solutions de l'équation: $\Delta u=\varphi u$, qui appartiennent à $L^{2}(\varphi)$ et dont les normes de Dirichlet classiques sont finies. D'après le théorème 2, on connaît que l'inclusion $C_{s}\left(N_{0}\right) \subset C_{s}\left(N_{\varphi}\right)$ n'a pas toujours lieu. 
Remarque 9. Soit $N_{0}$ un noyau régulier satisfaisant au principe complet du maximum; alors on a, pour une fonction $\varphi$ de $L_{\mathrm{loc}}^{+}(\xi)$ et pour un nombre $a$ avec $0 \leqq a \leqq 1, C_{s}\left(N_{0}\right) \cap C_{s}\left(N_{\varphi}\right) \subset C_{s}\left(N_{a_{\varphi}}\right)$.

En effet, soit $N^{\prime}$ un noyau singulier quelconque de $C_{s}\left(N_{0}\right) \cap C_{s}\left(N_{\varphi}\right)$; on pose respectivement $N=N_{0}+N^{\prime}, \tilde{N}_{\varphi}=N_{\varphi}+N^{\prime}$ et $\tilde{N}_{a \varphi}=N_{a \varphi}+N^{\prime}$. On a, quelles que soient $u$ de $H\left(N_{\varphi}\right) \cap L^{2}(\xi)$ et $v$ de $H\left(N^{\prime}\right)$,

$$
\begin{aligned}
& \|\inf (|u+v|, 1)\|_{H(N)}^{2}=\left\|u_{1}\right\|_{H\left(N_{0}\right)}^{2}+\left\|u_{2}\right\|_{H\left(N^{\prime}\right)}^{2} \leqq\|u+v\|_{H(N)}^{2}, \\
& \|\inf (|u+v|, 1)\|_{H\left(\tilde{N}_{\varphi}\right)}^{2}=\left\|u_{1}\right\|_{H\left(N_{\varphi}\right)}^{2}+\left\|u_{2}\right\|_{H\left(N^{\prime}\right)}^{2} \leqq\|u+v\|_{H\left(\tilde{N}_{\varphi}\right)}^{2},
\end{aligned}
$$

où $u_{1}$ et $u_{2}$ sont respectivement les projections de inf $(|u+v|, 1)$ sur $H\left(N_{0}\right)$ et sur $H\left(N^{\prime}\right)$. On a donc

$$
\begin{aligned}
\|\inf (|u+v|, 1)\|_{H\left(\tilde{N}_{a \varphi}\right)}^{2}= & a\left\|u_{1}\right\|_{H\left(N_{\varphi}\right)}^{2}+(1-a)\left\|u_{1}\right\|_{H\left(N_{0}\right)}^{2} \\
& +\left\|u_{2}\right\|_{H\left(N^{\prime}\right)}^{2} \leqq\|u+v\|_{H\left(\tilde{N}_{a \varphi}\right)}^{2},
\end{aligned}
$$

et par suite, $\tilde{N}_{a_{\varphi}}$ satisfait au principe complet du maximum, d'où $N^{\prime} \in C_{s}\left(N_{a \varphi}\right)$.

On obtient, de la même manière, $D_{s}\left(N_{0}\right) \cap D_{s}\left(N_{\varphi}\right) \subset D_{s}\left(N_{a \varphi}\right)$. Mais nous ne connaissons pas maintenant la comparaison explicite entre $C_{s}\left(N_{0}\right)$ et $C_{s}\left(N_{\varphi}\right)$. Elle sera peut-être utile pour déterminer $C_{s}\left(N_{0}\right)$. D'après la présente remarque, si $N_{0}$ est régulier et satisfait au principe complet du maximum (resp. au principe de domination), alors $C_{s}\left(N_{0}\right) \cap C_{s}\left(N_{p}\right) \subset$ $C_{s}\left(N_{q}\right)$ (resp. $D_{s}\left(N_{0}\right) \cap D_{s}\left(N_{p}\right) \subset D_{s}\left(N_{q}\right)$ ) dès que $q \leqq p$, où $\left(N_{p}\right)_{p>0}$ est la résolvante associée au noyau $N_{0}$.

\section{Les noyau singuliers satisfaisant au principe complet du maximum}

Pour un noyau $N$ relatif à $X$ et à $\xi$, on ne peut pas toujours le potentiel de la mesure de Dirac $\varepsilon_{x}$ à $x \in X$ par rapport au noyau $N$. Mais on peut considérer son support à un certain sens.

DÉFinition 6. Soient $N$ un noyau relatif à $X$ et à $\xi$, et $x$ un point de $X$; on pose, pour un système fondamental $\left(\omega_{n}\right)_{n=1}^{\infty}$ des voisinages de $x$,

$$
\operatorname{Supp}\left(N ; \varepsilon_{x}\right)=\bigcap_{n=1}^{\infty}\left\{x \in X ; N c_{\omega_{n}}(x)>0\right\},
$$

où $c_{\omega_{n}}$ est la fonction caractéristique de $\omega_{n}$. Cela est déterminé uniquement sauf l'ensemble de zéro $\xi$-mesure et s'appelle le support du $N$-potentiel de $\varepsilon_{x}$. 
Soient $f$ de $M_{K}^{+}$et $x$ un point de $X$; si, quel que soit $V$ un voisinage de $x, \int_{V} f d \xi>0$, on a évidemment $\operatorname{Supp}\left(N ; \varepsilon_{x}\right) \subset\{x \in X ; N f(x)>0\}$ sauf l'ensemble de zéro $\xi$-mesure.

Remarque 10. Soient $N_{0}$ un noyau régulier satisfaisant au principe de domination et $\left(N_{p}\right)_{p>0}$ la résolvante associée au noyau $N_{0}$. On a alors, quels que soient $p>0$ et $x$ de $X$,

$$
\xi\left(\operatorname{Supp}\left(N ; \varepsilon_{x}\right) \ominus \operatorname{Supp}\left(N_{p} ; \varepsilon_{x}\right)\right)=0,
$$

où $\ominus$ désigne la différence symétrique. On a, d'après l'équation résolvante,

$$
N_{0}=\sum_{n=1}^{\infty}(2 p)^{n-1}\left(N_{2 p}\right)^{n} \quad \text { et } \quad N_{p}=\sum_{n=1}^{\infty} p^{n-1}\left(N_{2 p}\right)^{n}
$$

Donc notre remarque en résulte immédiatement.

Remarque 11. Si un noyau régulier $N_{0}$ satisfait au principe de domination et s'il est partout dense dans $X \times X$, on a alors, pour tout $x$ de $X, \xi\left(X-\operatorname{Supp}\left(N_{0} ; \varepsilon_{x}\right)\right)=0$.

Pour un noyau régulier $N_{0}$ et pour une fonction $\varphi$ de $L_{\mathrm{loc}}^{+}(\xi), H_{\varphi}$ désigne l'espace fonctionnel $\left\{u \in H\left(N_{0}\right) ; \int|u(x)|^{2} \varphi(x) d \xi(x)<+\infty\right\}$ introduit le produit scalaire

$$
(u, v)_{H_{\varphi}}=\int u(x) v(x) \varphi(x) d \xi(x)+(u, v)_{H\left(N_{0}\right)} .
$$

Si $H_{\varphi}$ est à noyau positif, son noyau s'écrit aussi $N_{\varphi}$ et s'appelle le $\varphi$-noyau associé au noyau $N_{0}$.

THÉORÈME 4. Soient $N_{0}$ un noyau régulier et $N^{\prime}$ un noyau singulier avec $H\left(N_{0}\right) \cap H\left(N^{\prime}\right)=\{0\}$; supposons qu'il existe une fonction positive $\varphi$ de $L_{\text {loc }}^{+}(\xi)$ telle que, quel que soit $a \geqq 0$, le noyau $N_{a_{\varphi}}$ existe. Si, pour tout $a \geqq 0, N_{a \varphi}+N^{\prime}$ satisfait au principe complet du maximum, alors $N_{0}$ et $N^{\prime}$ satisfont au principe complet du maximum et, quelle que soit $u$ de $H\left(N^{\prime}\right)$, on a, pour $\xi$-presque tout $x$ de $X, u(y)=u(x) \xi-p . p$. sur $\operatorname{Supp}\left(N ; \varepsilon_{x}\right)$.

Si le présent théorème a lieu, alors, d'après la remarque 11 , on a le corollaire suivant: 
CorollaIre. Soit $N$ un noyau symétrique satisfaisant au principe complet du maximum. Si, quel que soit $p>0, N_{p}+N^{\prime}$ satisfait aussi au principe complet du maximum et si le support de $N_{0}$ est $X \times X$ sauf l'ensemble de zéro $\xi \times \xi$-mesure, $N$ s'exprime sous la forme $N=N_{0}+C$, où $C$ est une constante non-négative. Les notations $N_{0}, N^{\prime}$ et $\left(N_{p}\right)_{p>0}$ sont les mêmes que ci-dessus.

Pour montrer notre théorème, on préparera d'abord le lemme suivant:

LEMme 9. Soient $N$ un noyau symétrique satisfaisant au principe complet du maximum et $N^{\prime}$ sa part singulière; alors, pour une suite croissante $\left(K_{n}\right)_{n=1}^{\infty}$ de compacts de $X$ et pour un nombre $p>0$, on $a$, quelles que soient $f$ une fonction de $M_{K}$ et $u$ une fonction bornée de $H\left(N^{\prime}\right)$,

$$
\lim _{n \rightarrow+\infty} \iint f(x)(u(x)-u(y)) p N_{n, p}(d x, d y)=0,
$$

où $\left(N_{n, p}\right)_{p>0}$ est la résolvante sur $K_{n}$ associée au noyau $N+1$.

En effet, on peut supposer évidemment $C_{s}\left(N_{0}\right) \neq\{0\}$, et donc

$$
p N_{n, p} 1 \leqq 1 \text { et } \lim _{n \rightarrow+\infty} p N_{n, p} 1=1 \xi \text {-p.p. } \operatorname{sur} X \text {. }
$$

La fonction $u$ étant bornée, la présente intégrale a un sens. On a

$$
\begin{aligned}
& \varlimsup_{n \rightarrow+\infty}\left|\iint f(x)(u(x)-u(y)) p N_{n, p}(d x, d y)\right| \\
& \quad=\varlimsup_{n \rightarrow+\infty}\left|\iint f(x) u(x) p N_{n, p} 1(x) d \xi(x)-\left(u, N^{\prime}\left(p N_{n, p} f\right)\right)_{H\left(N^{\prime}\right)}\right|=0,
\end{aligned}
$$

car la suite $\left(N^{\prime}\left(p N_{n, p} f\right)\right)_{n=1}^{\infty}$ converge fortement vers $N^{\prime} f$ dans $H\left(N^{\prime}\right)$ avec $n \rightarrow+\infty$, d'où notre lemme.

Démonstration du théorème 4. On connaît déjà que $N_{0}$ satisfait au principe complet $\mathrm{du}$ maximum, et le principe complet du maximum pour $N^{\prime}$ est déduit de la même manière que dans l'implication (4) $\Rightarrow$ (1) dans le théorème 2 .

Montrons d'abord que si, pour une fonction $u$ de $H\left(N^{\prime}\right)$ et pour un ensemble $e$ de $E$, il existe une constante $c$ telle que $u(x)=c \xi$-p.p. sur $e$, alors on a, pour $\xi$-presque tout $x$ de $e, u(y)=c \xi$-p.p. $\operatorname{sur} \operatorname{Supp}\left(N ; \varepsilon_{x}\right)$. En effet, on peut supposer $e \in E_{0}, c \geqq 0$ et que $u$ est bornée sur $X$, car toute la contraction normale opère dans $H\left(N^{\prime}\right)$. On désigne par $c_{e}$ la 
fonction caractéristique de $e$, et on pose $v=\inf (u, c)$. Alors $v$ appartient aussi à $H\left(N^{\prime}\right)$. D'après le présent lemme, pour une suite croissante $\left(K_{n}\right)_{n=1}^{\infty}$ de compacts de $X$ avec $\bigcup_{n=1}^{\infty} K_{n}=X$,

$$
\lim _{n \rightarrow+\infty} \iint c_{e}(x)(v(x)-v(y)) p N_{n, p}(d x, d y)=0,
$$

où $\left(N_{n, p}\right)_{p>0}$ est la résolvante sur $K_{n}$ associée au noyau $N_{0}+N^{\prime}+1$. En utilisant le fait que, quelle que soit $f$ de $M_{K}, N_{n, p} f$ converge fortement vers $N_{p} f$ dans $L^{2}(\xi)$ avec $n \rightarrow+\infty$, où $\left(N_{p}\right)_{p>0}$ est la résolvante associée au noyau $N_{0}$, on obtient

$$
\begin{aligned}
0 & =\lim _{n \rightarrow+\infty} \iint c_{e}(x)(c-v(y)) p N_{n, p}(d x, d y) \\
& \geqq \iint c_{e}(x)(c-v(y)) p N_{p}(d x, d y) \geqq 0,
\end{aligned}
$$

car $c \geqq v \xi$-p.p. sur $X$. La fonction $v$ étant bornée, $p N_{p} v$ a un sens et on a

$$
p N_{p} v(x)=c p N_{p} 1(x) \xi-\text { p.p. sur } e,
$$

d'où, pour $\xi$-presque tout $x$ de $e, v(y)=c \xi$-p.p. $\operatorname{sur} \operatorname{Supp}\left(N ; \varepsilon_{x}\right)$. Par conséquent, pour $\xi$-presque tout $x$ de $e, u(y) \geqq c \xi$-p.p. $\operatorname{sur} \operatorname{Supp}\left(N ; \varepsilon_{x}\right)$. En répétant la même discution pour $u$, on a, pour $\xi$-presque tout $x$ de $e, u(y)=c \xi$-p.p. $\operatorname{sur} \operatorname{Supp}\left(N ; \varepsilon_{x}\right)$.

On remarque ici que, quelles que soient $u$ de $H\left(N^{\prime}\right)$ et $c$ une constante non-négative, $\sup (u, c)-c$ appartient à $H\left(N^{\prime}\right)$, car

$$
u=\inf (u, c)+(\sup (u, c)-c) .
$$

D'après la présente discussion, quelles que soient $u$ de $H\left(N^{\prime}\right)$ et $c_{1}, c_{2}$ deux constantes non-négatives avec $c_{1}<c_{2}$, on a, pour $\xi$-presque tout $x$ de

$$
e\left(u ; c_{1}, c_{2}\right)=\left\{x \in X ; c_{1} \leqq u(x) \leqq c_{2}\right\},
$$

$c_{1} \leqq u(y) \leqq c_{2} \quad \xi$-p.p. sur $\operatorname{Supp}\left(N ; \varepsilon_{x}\right)$. En effet, on peut supposer évidemment $\xi\left(e\left(u ; c_{1}, c_{2}\right)\right)>0$. Poser

$$
v_{1}=\inf \left(u, c_{1}\right) \text { et } v_{2}=\sup \left(u, c_{2}\right)-c_{2} \text {. }
$$

Alors $v_{1}=c_{1} \xi$-p.p. sur $e\left(u ; c_{1}, c_{2}\right)$ et $v_{2}=0 \xi$-p.p. sur $e\left(u ; c_{1}, c_{2}\right)$, et donc, pour $\xi$-presque tout $x$ de $e\left(u ; c_{1}, c_{2}\right), v_{1}(y)=c_{1} \xi$-p.p. $\operatorname{sur} \operatorname{Supp}\left(N ; \varepsilon_{x}\right)$ et $v_{2}(y)=0 \xi$-p.p. sur $\operatorname{Supp}\left(N ; \varepsilon_{x}\right)$, d'où, pour $\xi$-presque tout $x$ de $e\left(u ; c_{1}, c_{2}\right)$, $c_{1} \leqq u(y) \leqq c_{2} \xi$-p.p. $\operatorname{sur} \operatorname{Supp}\left(N ; \varepsilon_{x}\right)$. 
Soit $u$ une fonction non-négative de $H\left(N^{\prime}\right)$; posons

$$
\begin{aligned}
& e^{\prime}(u ; n)=\bigcup_{k=1}^{\infty}\left\{x \in X ; \frac{k}{n} \leqq u(x) \leqq \frac{k+1}{n},\right. \\
& \xi\left(\left\{y \in \operatorname{Supp}\left(N ; \varepsilon_{x}\right) ; u(y)>\frac{k+1}{n}\right.\right. \\
& \text { ou } \left.\left.\left.u(y)<\frac{k}{n}\right\}\right)>0\right\} .
\end{aligned}
$$

On a alors $\xi\left(e^{\prime}(u ; n)\right)=0$, d'où $\xi\left(\bigcup_{n=1}^{\infty} e^{\prime}(u ; n)\right)=0$. On a donc, pour $\xi$-presque tout $x$ de $X, u(y)=u(x) \xi$-p.p. $\operatorname{Supp}\left(N ; \varepsilon_{x}\right)$. Nous remarquons finalement que, pour une fonction $u$ de $H\left(N^{\prime}\right), u^{+}$et $u^{-}$appartiennent à $H\left(N^{\prime}\right)$, et nous achevons la démonstration.

Nous ne connaissons pas très bien l'inverse du présent théorème, mais le théorème suivant est proche son inverse.

THÉORÈmE 5. Soient $N_{0}$ un noyau régulier et $N^{\prime}$ un noyau singulier avec $H\left(N_{0}\right) \cap H\left(N^{\prime}\right)=\{0\}$. Si les trois conditions sont vérifiées, alors $N_{0}+N^{\prime}$ satisfait au principe complet du maximum.

(1) $N_{0}$ satisfait au principe complet du maximum.

(2) Quelle que soit $f$ de $M_{K}$, inf $\left(N^{\prime} f, 1\right)$ appartient à $H\left(N_{0}+N^{\prime}\right)$ et on $a$

$$
\left\|\inf \left(N^{\prime} f, 1\right)\right\|_{H\left(N_{0}+N^{\prime}\right)} \leqq\left(\int N^{\prime} f(x) f(x) d \xi(x)\right)^{1 / 2}
$$

(3) Quelle que soit $f$ de $M_{K}$, l'intégrale

$$
\iint N^{\prime} f(x)\left(N^{\prime} f(x)-N^{\prime} f(y)\right) p N_{p}(d x, d y)
$$

a un sens et est égale à 0 , où $\left(N_{p}\right)_{p>0}$ est la résolvante associée au noyau $N_{0}$.

Démonstration. Pour montrer que $N_{0}+N^{\prime}$ satisfait au principe complet du maximum, il suffit de voir que, quelle que soit $f$ de $M_{K}$, $\inf \left(N_{0} f+N^{\prime} f, 1\right)$ appartient à $H\left(N_{0}+N^{\prime}\right)$ et

$$
\left\|\inf \left(N_{0} f+N^{\prime} f, 1\right)\right\|_{H\left(N_{0}+N^{\prime}\right)} \leqq\left(\int\left(N_{0} f+N^{\prime} f\right) f d \xi\right)^{1 / 2} .
$$

En effet, si, l'on admet le présent énoncé, de la manière usuelle dans cet article, quelle que soit $u$ de $H\left(N_{0}+N^{\prime}\right)$, inf $(u, 1)$ appartient aussi à 
$H\left(N_{0}+N^{\prime}\right)$ et sa norme dans $H\left(N_{0}+N^{\prime}\right)$ est $\leqq\|u\|_{H\left(N_{0}+N^{\prime}\right)}$. On a donc, pour tout entier positif $n,(1 / n) \inf (n u, 1) \in H\left(N_{0}+N^{\prime}\right)$ et

$$
\left\|\frac{1}{n} \inf (n u, 1)\right\|_{H\left(N_{0}+N^{\prime}\right)} \leqq\|u\|_{H\left(N_{0}+N^{\prime}\right)} .
$$

Faisant $n \rightarrow+\infty$, on a $u^{+} \in H\left(N_{0}+N^{\prime}\right)$, et la norme de $u^{+}$dans $H\left(N_{0}+N^{\prime}\right)$ est plus petite que celle de $u$. Par conséquent, la contraction d'unité opère dans $H\left(N_{0}+N^{\prime}\right)$.

Pour une fonction $f$ de $M_{K}$ et pour un nombre $p>0$, on pose

$$
u_{p, f}=\inf \left(N_{p} f+N^{\prime} f, 1\right)-\inf \left(N^{\prime} f, 1\right),
$$

et alors $\left|u_{p, f}\right| \leqq\left|N_{p} f\right| \in L^{2}(\xi)$. Donc, quel que soit $q>0, N_{q} u_{p, f}$ a un sens et on a

$$
\begin{aligned}
q \iint u_{p, f}(x)\left(u_{p, f}(x)-\right. & \left.u_{p, f}(y)\right)\left(q N_{q}\right)(d x, d y) \\
=q\left(\int\left|u_{p, f}(x)\right|^{2}(1\right. & \left.-q N_{q} 1(x)\right) d \xi(x) \\
& \left.+\frac{1}{2} \iint\left(u_{p, f}(x)-u_{p, f}(y)\right)^{2}\left(q N_{q}\right)(d x, d y)\right) .
\end{aligned}
$$

On a, pour $\xi$-presque tous $x, y$ de $X$,

$$
\left|u_{p, f}(x)-u_{p, f}(y)\right| \leqq\left|N_{p} f(x)-N_{p} f(y)\right|+2\left|N^{\prime} f(x)-N^{\prime} f(y)\right|,
$$

et d'après la condition (3),

$$
\iint\left(N^{\prime} f(x)-N^{\prime} f(y)\right)^{2}\left(q N_{q}\right)(d x, d y)=0
$$

Done on a

$$
\begin{aligned}
& q \iint u_{p, f}(x)\left(u_{p, f}(x)-u_{p, f}(y)\right)\left(q N_{q}\right)(d x, d y) \\
& \quad \leqq q\left(\int\left|N_{p} f(x)\right|^{2}\left(1-q N_{q} 1(x)\right)+\frac{1}{2} \iint\left|N_{p} f(x)-N_{p} f(y)\right|^{2}\left(q N_{q}\right)(d x, d y)\right) \\
& \quad=q \iint N_{p} f(x)\left(N_{p} f(x)-N_{p} f(y)\right)\left(q N_{q}\right)(d x, d y)=\left\|N_{p} f\right\|_{H\left(N_{0}+(1 / q) U\right)}^{2},
\end{aligned}
$$

d'où

$$
\left\|u_{p, f}\right\|_{H\left(N_{0}+(1 / q) U\right)} \leqq\left\|N_{p} f\right\|_{H\left(N_{0}+(1 / q) U\right)} .
$$

Faisant $q \rightarrow+\infty$, on obtient que $u_{p, f}$ appartient à $H\left(N_{0}\right)$ et on a 


$$
\left\|u_{p, f}\right\|_{H\left(N_{0}\right)} \leqq\left\|N_{p} f\right\|_{H\left(N_{0}\right)},
$$

et ensuite, faisant $p \rightarrow 0$, on a inf $\left(N_{0} f+N^{\prime} f, 1\right)-\inf \left(N^{\prime} f, 1\right) \in H\left(N_{0}\right)$ et

$$
\left\|\inf \left(N_{0} f+N^{\prime} f, 1\right)-\inf \left(N^{\prime} f, 1\right)\right\|_{H\left(N_{0}\right)} \leqq\left\|N_{0} f\right\|_{H\left(N_{0}\right)} .
$$

D'après la condition (2), inf $\left(N^{\prime} f, 1\right)$ appartient à $H\left(N_{0}+N^{\prime}\right)$, et donc $\inf \left(N_{0} f+N^{\prime} f, 1\right)$ appartient aussi à $H\left(N_{0}+N^{\prime}\right)$. On a, de plus,

$$
\begin{aligned}
\| \inf ( & \left.N_{0} f+N^{\prime} f, 1\right)\left\|_{H\left(N_{0}+N^{\prime}\right)}^{2} \leqq\right\| \inf \left(N_{0} f+N^{\prime} f, 1\right)-\inf \left(N^{\prime} f, 1\right) \|_{H\left(N_{0}\right)}^{2} \\
& +\left\|\inf \left(N^{\prime} f, 1\right)\right\|_{H\left(N_{0}+N^{\prime}\right)}^{2} \leqq\left\|N_{0} f\right\|_{H\left(N_{0}\right)}^{2}+\left\|N^{\prime} f\right\|_{H\left(N^{\prime}\right)}^{2} \\
= & \left\|N_{0} f+N^{\prime} f\right\|_{H\left(N_{0}+N^{\prime}\right)}^{2},
\end{aligned}
$$

d'où $N_{0}+N^{\prime}$ satisfait au principe complet du maximum. La démonstration est ainsi complète.

Si $N^{\prime}$ satisfait au principe complet du maximum, la condition (2) est évidemment vérifiée. Mais son inverse n'a pas toujours lieu.

Remarque 12. Dans le présent théorème, si $N_{0}$ vérifie la condition que, quelles que soient $f, g$ de $M_{K}$,

$$
\iint N_{0} f(x) g(x) d \xi(x)=\int g(x) \int_{\operatorname{Supp}\left(N_{0} ; \varepsilon_{x}\right)} f(y) N_{0}(d x, d y),
$$

alors la conclusion du théorème 4 déduit la condition (3). Si $N_{0}$ est égal à une fonction localement $\xi \times \xi$-sommable et non-négative en dehors de l'ensemble diagonal de $X \times X$, la condition notée ci-dessus est vérifiée.

\section{Appendices sur les noyaux réguliers}

$1^{\circ}$ Les noyaux continus

Soit $G(x, y)$ une fonction continue au sens large, non-négative et symétrique dans $X \times X$; supposons ensuite $G(x, x)>0$ pour tout $x$ de $X$ et que, quel que soit $\omega$ un ouvert non-vide de $X$, il existe une mesure de Radon positive $\nu_{\omega} \neq 0$ à support dans $\omega$ telle que la $G$-énergie de $\nu_{\omega}, \int G \nu_{\omega} d \nu_{\omega}=\iint G(x, y) d \nu_{\omega}(y) d \nu_{\omega}(x)$, soit finie. Si le noyau-fonction continue $G$ satisfait au principe complet du maximum ${ }^{(4)}$, alors il existe une mesure de Radon positive $\xi$ partout dense dans $X$ telle que le noyau

(4) Cela signifie que, pour deux mesures de Radon positives $\mu, \nu$ dans $X$ à support compact et de $G$-énergie finie, $G \mu(x) \leqq G \nu(x)+1$ sur $\operatorname{Supp}(\mu) \Rightarrow G \mu(x) \leqq G \nu(x)+1$ sur $X$, où $G \mu(x)=\int G(x, y) d \mu(y)$. 


$$
N\left(e_{1}, e_{2}\right)=\int_{e_{1}} \int_{e_{2}} G(x, y) d \xi(y) d \xi(x)
$$

relatif à $X$ et à $\xi$ soit régulier et satisfasse au principe complet $d u$ maximum.

En effet, $X$ étant à base dénombrable, on peut choisir une base dénombrable $\left(\omega_{n}\right)_{n=1}^{\infty}$ d'ouverts de $X$. D'après notre condition, il existe une mesure de Radon positive $\nu_{n}(\neq 0)$ à support compact dans $\omega_{n}$ telle que $\int G \nu_{n} d \nu_{n}<+\infty$. D'après le principe complet du maximum pour $G$, on peut supposer que le $G$-potentiel de $\nu_{n}$,

$$
G \nu_{n}(x)=\int G(x, y) d \nu_{n}(y)
$$

est borné sur $X$. On choit une suite $\left(a_{n}\right)_{n=1}^{\infty}$ de nombres positifs telle que $\sum_{n=1}^{\infty} a_{n} \int d \nu_{n}<+\infty$, et on pose $\xi=\sum_{n=1}^{\infty} a_{n} \nu_{n}$. Posons

$$
N\left(e_{1}, e_{2}\right)=\int_{e_{1}} \int_{e_{2}} G(x, y) d \xi(y) d \xi(x) ;
$$

alors $N$ est un noyau symétrique relatif à $X$ et à $\xi$ et satisfait au principe complet du maximum. D'après la proposition 2 et $H(N+U)$ $\ni 1, N$ est régulier.

$2^{\circ}$ Noyaux de convolution symétriques

Lorsque $X$ est un groupe abélien et $\xi$ est sa mesure de Haar, un noyau $N$ relatif à $X$ et à $\xi$ s'appelle un noyau de convolution sur $X$ s'il existe une mesure de Radon positive $\kappa$ dans $X$ telle que, quels que soient $e_{1}, e_{2}$ de $E$,

$$
N\left(e_{1}, e_{2}\right)=\int c_{1} * \check{c}_{2}(x) d \kappa(x),
$$

où $c_{i}(i=1,2)$ est la fonction caractéristique de $e_{i}$ et $\check{c}_{2}(x)=c_{2}(-x)$. Dans ce cas, $\kappa$ est uniquement déterminée, et elle s'appelle aussi un noyau de convolution sur $X$. Si $N$ est symétrique si et seulement si $\kappa$ est symétrique par rapport à l'origine de $X$.

Remarque 13. Si un noyau de convolution symétrique $\kappa$ sur $X$ satisfait au principe de domination et s'il est régulier à notre sens, alors $H(\kappa) \cap C_{K}$ est dense dans $H(\kappa)$, où $C_{K}$ est l'espace des fonctions finies et continues dans $X$ à support compact, et muni de la topologie usuelle.

On remarque d'abord que, pour un noyau de convolution symétrique sur $X$, le principe de domination et le principe complet du maximum 
sont équivalents. On a, quelles que soient $f \geqq 0$ de $C_{K}$ et $u$ de $H(\kappa)$, $u * f \in H(\kappa)$ et

$$
\|u * f\|_{H(\kappa)} \leqq\left(\int f d \xi\right)\|u\|_{H(\kappa)} .
$$

$H(\kappa) \cap L^{2}(\xi)$ étant dense dans $H(\xi), C_{0} \cap H(\kappa)$ est dense dans $H(\kappa)$, où $C_{0}$ est l'espace des fonctions finies et continues dans $X$ s'annulant à l'infini. Pour une fonction $u \geqq 0$ de $C_{0} \cap H(\kappa)$, sup $(u, 1 / n)-1 / n$ appartient à $C_{K} \cap H(\kappa)$ et elle converge fortement vers $u$ dans $H(\kappa)$ avec $n \rightarrow+\infty$, d'où $H(\kappa) \cap C_{K}$ est dense dans $H(\kappa)$.

Remarque 14. Soit $\kappa_{0}$ un noyau de convolution régulier à notre sens ; alors, quel que soit $\kappa^{\prime}$ de $D_{s}\left(\kappa_{0}\right)$, on a $C_{0} \cap H\left(\kappa^{\prime}\right)=\{0\}$ et $\kappa^{\prime}$ satisfait au principe complet du maximum.

En effet, soit $u$ une fonction de $C_{0} \cap H\left(\kappa^{\prime}\right) . \quad \kappa_{0}+\kappa^{\prime}$ satisfaisant au principe complet du maximum, $\sup (u, 1 / n)-1 / n$ appartient à $C_{K} \cap$ $H\left(\kappa_{0}+\kappa^{\prime}\right)$, d'où $\sup (u, 1 / n)-1 / n \in H\left(\kappa_{0}\right)$. Faisant $n \rightarrow+\infty$, on a $u \in$ $H\left(\kappa_{0}\right)$, d'où $u=0$. Ayant $\kappa^{\prime} \in D_{s}\left(\kappa_{0}+\varepsilon\right)$, où $\varepsilon$ est la mesure de Dirac à l'origine, $\kappa^{\prime}$ satisfait au principe complet du maximum, en utilisant le théorème de [6] et la remarque 13 .

De plus, si un noyau de convolution $\kappa^{\prime}$ à notre sens satisfait au principe complet du maximum, $C_{0} \cap H\left(\kappa^{\prime}\right)=\{0\}$, car $\kappa^{\prime} \in D_{s}(\varepsilon)$.

\section{REFERENCES}

[1] A. Beurling et J. Deny: Dirichlet spaces, Proc. Nat. Acad. Sci. U.S.A., 45, $1959,208-215$.

[2] G. Choquet et J. Deny: Aspects linéaires de la théorie du potentiel III, C. R. Acad. Sci. Paris, 243, 1960, 4260-4262.

[ 3 ] J. Deny: Principe complet du maximum et contractions, Ann. Inst. Fourier, 15, 1965, 259-271.

[4] M. Itô: Note sur contractions et principes du maximum, Osaka Math. J., 4, 1967, 217-226.

[5] _ - Sur les noyaux d'ordre fractionnaire associés au noyau de Dirichlet, Hiroshima Math. J., 1, 1971, 123-143.

[6] —-: Noyaux de convolution réguliers et noyaux de convolution singuliers, Nagoya Math. J., 44, 1971, 61-77.

[ 7 ] 44, 1971, 133-164.

[ 8 ] M. Kishi: General theory of potentiel, Lecture note, 1970/71, Nagoya Univ. 